\title{
A novel picture fuzzy linguistic Muirhead Mean aggregation operators and their application to multiple attribute decision making
}

\author{
Sidong Xian ${ }^{1}\left[\right.$ : $\cdot$ Yue Cheng ${ }^{1} \cdot$ Zhou Liu $^{2}$ \\ Accepted: 4 August 2021 / Published online: 20 August 2021 \\ ๑) The Author(s), under exclusive licence to Springer-Verlag GmbH Germany, part of Springer Nature 2021
}

\begin{abstract}
The Picture fuzzy linguistic set (PFLS) is an extension of the intuitionistic fuzzy set (IFS) and linguistic variables (LVs), which has been applied successfully in the process of decision making. Considering the lack of closeness of extant PFLS operations and the interrelationship among input attributes do not consider. In this paper, for the sake of addressing those limitations, we firstly redefine some novel operational laws for PFLS by introducing linguistic scale functions and the related properties are studied. Then, a novel score function and accuracy function are also defined to compare PFLSs. Subsequently, in consideration of the superiority of the Muirhead Mean (MM) operator in capturing the interaction relationship between the input parameters, we extend the MM operator to the Picture fuzzy linguistic context and then propose Picture fuzzy linguistic weighted MM operator and its dual form in a new light. After that, these operators have adopted to build two novel models to solve multiple attribute decision-making (MADM) problems. Finally, a practical example for the selection of the innovative "Mobike" sharing bike design is provided to illustrate the practicality and effectiveness of proposed approaches.
\end{abstract}

Keywords Picture fuzzy linguistic set $\cdot$ Muirhead Mean (MM) operator $\cdot$ Linguistic scale functions $\cdot$ Multiple attribute decision making

\section{Introduction}

Decision making (DM) is a common activity in our daily life. Owing to the DM problems are usually uncertain and fuzzy, it is difficult for decision makers (DMs) to depict the attribute values as real numbers. Hence, Zadeh (1965) proposed the concept of the linguistic variable and fuzzy set (FS) to process the fuzzy linguistic information. Afterward, based on fuzzy set theory, intuitionistic fuzzy set (IFS) was first introduced by Atanassov (1986), which can be used to describe uncertainty more comprehensively than FS, and attracted many researchers' attention Singh et al. (2020); Wang (2020); Akram et al. (2021).

Sidong Xian

sidx@163.com

1 Key Laboratory of Intelligent Analysis and Decision on Complex Systems, Chongqing University of Posts and Telecommunications, Chongqing 400065, People's Republic of China

2 School of automation, Chongqing University of Posts and Telecommunications, Chongqing 400065, People's Republic of China
Although the IFS theory has been obtained many achievements in practical application, there are some situations in which it is inappropriate to handle the information combined with IFS. Voting is a typical example, generally, people's voting views contain multiple types, including vote support, vote again, abstain and refuse to vote, which cannot be accurately represented by traditional FS or IFS. Therefore, to overcome such issues, Cuong and Kreinovich (2014) originated the notion of the Picture fuzzy set (PFS), which is a generalization of the IFS. The Picture fuzzy set is composed of three degrees representing the degree of positive membership, the degree of neutral membership, and the degree of negative membership. Since the appearance of the PFS, much progress has been made in the research of the PFS theory Cuong and Hai (2015); Singh (2015); Son (2015); Zhang et al. (2020); Ju et al. (2020); Yang et al. (2020); Wang et al. (2020); Qiyas et al. (2020); Jana et al. (2019); Wei (2018); Khalil et al. (2019). Such as Singh (2015) put forward a clustering analysis method based on his research on the correlation coefficients of PFS. Jana et al. Jana et al. (2019) extend the Dombi operator to the PFS domain and proposed a variety of aggregation operators to solve picture 
fuzzy MADM problems. Khalil et al. (2019) put forward an interval-valued Picture fuzzy soft set and applied it to the data during analysis. Si et al. (2021) proposed a decision-making method for optimal drug selection using Picture Fuzzy Set (PFS), Dempster-Shafer (D-S) Evidence Theory and Grey Relational Analysis (GRA) to provide better treatment for COVID-19 patients.

It is clear to find that in the above multiple information representation tools, the performance of each alternative under different criteria is by experts based on their knowledge and experience for DM problems in quantitative form. However, due to the nature of qualitative criteria and uncertainty of the real problems, DMs prefer to use linguistic terms (LTs) to express their opinions. Though the linguistic terms are not as good as numbers in terms of information representation accuracy, they are closer to human linguistic habit. For example, when people evaluate the risk level of the stock market, the linguistic term "low", "medium" and "high" can be employed, which are more in line with the human cognitive process. Up to now, a lot of extended fuzzy linguistic approaches have been developed. Combing IFS theory with linguistic variables, Li and Wang (2009) proposed an intuitionistic linguistic set (ILS). Xian et al. (2015) developed the intuitionistic fuzzy linguistic-induced ordered weighted averaging (IFLIOWA) operator, which attributes values in the form of intuitionistic fuzzy linguistic variables. More recently, based on PFS and linguistic variables, Peng and Yang (2016) proposed the Pythagorean fuzzy linguistic sets (PFLSs) and applied it to the evaluation of emergency response capabilities of the government departments. Akram et al. (2021) developed a hesitation fuzzy N soft ELECREII method to adapt to the hesitation in the MADM problem. Inspired by the idea of Lq-ROFS, Akram et al. (2021) uses the flexible characteristics of Einstein operators to introduce language q-rung orthopair fuzzy graphs (Lq-ROFGs) to further explore effective methods for handling complex MAGDM situations. Adeel proposed Adeel et al. (2019) a new concept of $m$-polar fuzzy linguistic variables ( $m$ FLV) to increase the richness of linguistic variables based on m-polar fuzzy $(m \mathrm{~F})$ methods, on this basis, has also developed a method Adeel et al. (2019) for multi-criteria group decision making (MCGDM) the $m$-polar fuzzy language TOPSIS method.

In view of the superiority of LTs in facilitating the expression of evaluation values by DMs, at the same time, considering that there is little research on extending linguistic variables to PFS. Ashraf et al. (2018) proposed the concept of picture fuzzy linguistic set on the basis of PFS and LTs to describe the complex cognitive information, and the corresponding operational laws of PFLS are defined. Meanwhile, by introducing Archimedean triangular norms (t-norms) and triangular conorms (s-norms), Liu and Zhang (2018) defined new operational rules for PFLVs to handle cases concerning the selection of ERP system. However, because PFLS has not appeared for a long time, the comparison rules and operational rules are not perfect, there are situations that exceed the defined limits or cannot be compared. Take the MADM problem of the research sexual treatment plan for COVID-19 patients Si et al. (2021) as an example, suppose that $S=\left\{s_{0}, s_{1}, \ldots, s_{6}\right\}$ be a LTS, $H_{1}=\left\langle\mathrm{s}_{3}, 0.3,0.2,0.5\right\rangle$ and $H_{2}=\left\langle\mathrm{s}_{5}, 0.2,0.4,0.3\right\rangle$ are two PFLSs, then by the operational rules given by Ashraf et al. (2018), we can obtain the result of $H_{1} \oplus H_{2}$ is $\left\langle s_{8}, 0.44,0.08,0.15\right\rangle$, obviously, the linguistic term part beyond the upper bound of redefined $\mathrm{S}$. This seems unreasonable. Assume that $H_{1}=\left\langle\mathrm{s}_{3}, 0.5,0,0.3\right\rangle$ and $H_{2}=\left\langle\mathrm{s}_{3}, 0.3,0,0.1\right\rangle$ are two PFLSs, we can obtain $H_{1}$ is indifferent from $\mathrm{H}_{2}$, which does not totally meet our intuition. Moreover, the existing MADM problems are often dealt with without considering the correlation between attributes. Muirhead Mean (MM) operator was first proposed by Muirhead (1902), which is a powerful and useful aggregation operator in information fusion. Compared with the existing commonly used operators with the same function, such as BM Gou et al. (2017); Xia et al. (2013) or MSM liu et al. (2020); Rong et al. (2020) operators, the prominent advantage of the MM is that it can consider the interrelationship among any input arguments by an alterable parameter, which can apply in different application scenarios. Moreover, BM and MSM operators are special cases of MM operators. In this sense, the MM operator can get more comprehensive fusion information than BM or MSM operator. What's more, to the best of our knowledge, there is a research gap concerning the MM operator with the evaluation information expressed in PFLVs. Thus, it is necessary to extend the MM operator to the field of Picture fuzzy linguistic and discussed some special properties of the developed operators. Then, with the purpose of more clearly expressing the intent of this paper, the specific motivation is as follows:

- The extant operational rules of PFLSs are not closed. Because in some special cases, the linguistic term part might beyond the upper bound of redefined $S$. This paper redefines some novel operational laws for PFLS by introducing linguistic scale functions (LSFs), which can effectively make up the flaws of existing operational laws.

- The comparison rules of PFLSs cannot work in some cases and is hard to explain the meaning of subscript operations in linguistic terms, especially multiplication and division. A novel score function and accuracy function are proposed, and corresponding comparison rules of PFLVs are defined, which can improve the comparison rules.

- In real MADM cases, the attributes tend to interact with each other. However, the vast majority of current studies assume that attributes are independent. The MM operator is extended and its dual form to the Picture fuzzy linguis- 
tic environment and develop two aggregation operators in this paper, which can capture the interrelationship of attributes to make the results more reasonable.

To do so, the remainder of this paper is structured as follows: In Sect. 2, briefly review some concepts of LTS, PFLS, MM, and DMM operators. In Sect. 3, redefined some novel operational laws of PFLVs by introducing the LSFs, and the corresponding comparison rules are developed. In Sect. 4, combine the MM operator and its dual form with picture fuzzy linguistic information and put forward picture fuzzy linguistic aggregation operators, including picture fuzzy linguistic weighted MM (PFLWMM) operator and picture fuzzy linguistic weighted DMM (PFLWDMM) operator, and the properties of them are studied. In the next section, utilize those operators to create two models to solve MADM problems under picture fuzzy linguistic context. In Sect. 6, a case study about the selection of the innovative "Mobike" sharing bike design is provided to illustrate the usefulness and practicality of our proposed methods, and the conclusion is included in Sect. 7.

\section{Preliminaries}

In the following, we briefly recall some concepts and properties of linguistic term set (LTS), Picture fuzzy set (PFS), Picture fuzzy linguistic set (PFLS), and Muirhead Mean (MM) operators.

\subsection{The linguistic term set}

Suppose that $S=\left\{s_{t} \mid t=0,1, \ldots, l-1\right\}$ be a linguistic term set (LTS) with odd cardinality, where $s_{t}$ represents possible value for a linguistic variable. In addition, the linguistic elements in $S$ should satisfy the following conditions Herrera et al. (1995); Zadeh (1975):

1) If $s_{a}, s_{b} \in S$ and $a<b$, then $s_{a}<s_{b}$;

2) There exists the negation operator: $n e g\left(s_{a}\right)=s_{b}$, where $a=l-1-\mathrm{b}$

3) $\operatorname{Max}\left(s_{a}, s_{b}\right)=s_{a}$, if $s_{a} \geq s_{b}$;

4) $\operatorname{Min}\left(s_{a}, s_{b}\right)=s_{a}$, if $s_{a} \leq s_{b}$.

For example, when $l=7$, then $S$ could be given as follows: $S=\left\{s_{0}=\right.$ extremely poor, $s_{1}=$ poor, $s_{2}=$ slight poor, $s_{3}=$ fair, $s_{4}=$ slight good, $s_{5}=$ good, $s_{6}$ $=$ very good .

Furthermore, in order to retain original decision information as much as possible, $\mathrm{Xu}$ (2004) extended the discrete linguistic term set to a continuous form $\bar{S}=\left\{s_{t} \mid t \in[0, l-\right.$ $1]\}$, where $l$ is a sufficiently large positive integer.

\subsection{Picture fuzzy set}

Definition 1 Cuong and Kreinovich (2014) Let $X$ be a fixed finite set. Then, the Picture fuzzy set (PFS) $A$ on $X$ is proposed by Cuong and Kreinovich (2014) as follows:

$A=\left\{\left\langle x, P_{A}(x), I_{A}(x), N_{A}(x)\right\rangle \mid x \in X\right\}$.

where the functions $P_{A}(x): X \rightarrow[0,1] ; I_{A}(x):$ $X \rightarrow[0,1] ; N_{A}(x): X \rightarrow[0,1]$ with the following condition $0 \leq P_{A}(x)+I_{A}(x)+N_{A}(x) \leq 1, \forall x \in$ $X$. The functions $P_{A}(x), I_{A}(x)$, and $N_{A}(x)$, respectively, represent positive-membership degree, neutral-membership degree and negative-membership degree of element $x$ in $X$. The degree of refusal-membership is defined for $x$ as $\pi_{A}(x)=1-P_{A}(x)-I_{A}(x)-N_{A}(x)$. For convenience, the pair $\left(P_{A}(x), I_{A}(x), N_{A}(x)\right)$ is called Picture fuzzy number (PFN).

Furthermore, Cuong and Kreinovich (2014) defined some basic logical operations of PFN, which are shown as follows:

Definition 2 Cuong and Hai (2015) Let $A=\left(P_{A}(x), I_{A}(x)\right.$, $\left.N_{A}(x)\right)$ and $B=\left(P_{B}(x), I_{B}(x), N_{B}(x)\right)$ be any two PFNs over the universe $X$. Then the operations between two PFNs are stated as:

(1) If $P_{A}(x) \leq P_{B}(x), I_{A}(x) \geq I_{B}(x)$ and $N_{A}(x) \geq N_{B}(x)$ for all $x \in X$, then $A \subseteq B$;

(2) $A=B$ if $A \subseteq B$ and $B \subseteq A$;

(3) $A \cup B=\left\{\left\langle x, \max \left\{P_{A}(x), P_{B}(s)\right\}, \min \left\{I_{A}(x), I_{B}(x)\right\}\right.\right.$, $\left.\left.\min \left\{N_{A}(x), N_{B}(x)\right\}\right\rangle \mid x \in X\right\}$

(4) $A \cap B=\left\{\left\langle x, \min \left\{P_{A}(x), P_{B}(s)\right\}, \max \left\{I_{A}(x), I_{B}(x)\right\}\right.\right.$, $\left.\left.\max \left\{N_{A}(x), N_{B}(x)\right\}\right\rangle \mid x \in X\right\}$.

Subsequently, Wei Cuong and Hai (2015) constructed some novel operations of PFN on the basis of the operation rules of IFS as follows:

Definition 3 Wei (2017) Let $A=\left(P_{A}(x), I_{A}(x), N_{A}(x)\right)$ and $B=\left(P_{B}(x), I_{B}(x), N_{B}(x)\right)$ be any two PFNs over the universe $X, \lambda \in[0,1]$. Then the operational laws are defined as:

(1) $A \oplus B=\left(P_{A}(x)+P_{B}(x)-P_{A}(x) P_{B}(x), I_{A}(x) I_{B}(x)\right.$, $\left.N_{A}(x) N_{B}(x)\right)$

(2) $A \otimes B=\left(P_{A}(x) P_{B}(x), I_{A}(x)+I_{B}(x)-I_{A}(x) I_{B}(x)\right.$, $\left.N_{A}(x)+N_{B}(x)-N_{A}(x) N_{B}(x)\right)$

(3) $\lambda A=\left(1-\left(1-P_{A}(x)\right)^{\lambda}, I_{A}(x)^{\lambda}, N_{A}(x)^{\lambda}\right)$;

(4) $A^{\lambda}=\left(P_{A}(x)^{\lambda}, 1-\left(1-I_{A}(x)\right)^{\lambda}, 1-\left(1-N_{A}(x)\right)^{\lambda}\right)$;

(5) $A^{C}=\left(N_{A}(x), I_{A}(x), P_{A}(x)\right)$. 


\subsection{Picture fuzzy linguistic set}

Definition 4 Ashraf et al. (2018) Let $X$ be a fixed universe, and $S=\left\{s_{0}, s_{1}, \ldots, s_{\tau-1}\right\}$ be a LTS. Then, the Picture fuzzy linguistic set $H$ in $X$ is denoted as follows:

$H=\left\{\left\langle s_{\theta(x)}, P_{H}(x), I_{H}(x), N_{H}(x)\right\rangle \mid x \in X\right\}$.

where $s_{\theta(x)} \in S$, the numbers $P_{H}(x), I_{H}(x)$ and $N_{H}(x)$, respectively, represent positive membership degree, neutral membership degree and negative membership degree of element $x$ to $s_{\theta(x)}$, and satisfy $P_{H}(x), I_{H}(x), N_{H}(x) \in[0,1]$ and $0 \leq P_{H}(x)+I_{H}(x)+N_{H}(x) \leq 1$. For $\forall x \in X, \pi_{H}(x)=$ $1-P_{H}(x)-I_{H}(x)-N_{H}(x)$ could be represented the refusal degree of element $x$ to $s_{\theta}(x)$. For convenience, the simplification of $H$ is denoted by $\left\langle s_{\theta(x)},(P(x), I(x), N(x))\right\rangle$, which is called Picture fuzzy linguistic variable (PFLV).

At the same time, Ashraf et al. (2018) defined some operational laws of PFLV as follows:

Definition 5 Ashraf et al. (2018) Let $H_{i}=\left\langle s_{\theta\left(x_{i}\right)},\left(P\left(x_{i}\right)\right.\right.$, $\left.\left.I\left(x_{i}\right), N\left(x_{i}\right)\right)\right\rangle(i=1,2)$ be any two PFLVs and $\lambda \geq 0$. Then the operations of PFLV can be denoted as:

(1) $\lambda H_{1}=\left\langle s_{\lambda \theta\left(x_{1}\right)},\left(1-\left(1-P\left(x_{1}\right)\right)^{\lambda}, I\left(x_{1}\right)^{\lambda}, N\left(x_{1}\right)^{\lambda}\right)\right\rangle$;

(2) $H_{1}^{\lambda}=\left\langle s_{\left(\theta\left(x_{1}\right)\right)^{\lambda}},\left(P\left(x_{1}\right)^{\lambda}, 1-\left(1-I\left(x_{1}\right)\right)^{\lambda}, 1-(1-N\right.\right.$ $\left.\left.\left.\left(x_{1}\right)\right)^{\lambda}\right)\right\rangle$

(3) $H_{1} \oplus H_{2}=\left\langle s_{\theta\left(x_{1}\right)+\theta\left(x_{2}\right)},\left(P\left(x_{1}\right)+P\left(x_{2}\right)-P\left(x_{1}\right) P\left(x_{2}\right)\right.\right.$, $\left.\left.I\left(x_{1}\right) I\left(x_{2}\right), N\left(x_{1}\right) N\left(x_{2}\right)\right)\right\rangle$;

(4) $H_{1} \otimes H_{2}=\left\langle s_{\theta\left(x_{1}\right) \times \theta\left(x_{2}\right)},\left(P\left(x_{1}\right) P\left(x_{2}\right), I\left(x_{1}\right)+I\left(x_{2}\right)-\right.\right.$ $\left.\left.I\left(x_{1}\right) I\left(x_{2}\right), N\left(x_{1}\right)+N\left(x_{2}\right)-N\left(x_{1}\right) N\left(x_{2}\right)\right)\right\rangle$.

\subsection{MM operator}

The MM operator was originally introduced by Murihead Muirhead (1902).

Definition 6 Muirhead (1902) Assume that $a_{j}(j=1,2, \ldots$, $n$ ) be a group of non-negative real numbers, and $Q=$ $\left(Q_{1}, Q_{2}, \ldots, Q_{n}\right) \in R^{n}$ be a vector of parameters. Then MM operator is explained as

$M M^{Q}\left(a_{1}, a_{2}, \ldots, a_{n}\right)=\left(\frac{1}{n !} \sum_{\sigma \in S_{n}} \prod_{j=1}^{n} a_{\sigma(j)}^{Q_{j}}\right)^{\frac{1}{\sum_{j=1}^{n} Q_{j}}}$.

Furthermore, then dual MM (DMM) operator is proposed by liu et al. (2020) as follows:

$$
\begin{aligned}
& \operatorname{DMM}^{Q}\left(a_{1}, a_{2}, \ldots, a_{n}\right) \\
& =\frac{1}{\sum_{j=1}^{n} Q_{j}}\left(\sum_{\sigma \in S_{n}} \prod_{j=1}^{n} Q_{j} a_{\sigma(j)}\right)^{\frac{1}{n !}} \text {. }
\end{aligned}
$$

where $\sigma(j)(j=1,2, \ldots, n)$ is any permutation of $\{1,2, \ldots$, $n\}$ and $S_{n}$ is the set of all permutations of $\{1,2, \ldots, n\}$.

\section{Some novel operational laws and measures for PFLS}

\subsection{Linguistic scale functions}

Traditional operational laws of PFLS are to calculate directly utilizing the subscript of linguistic terms based on assumptions that the deviation between adjacent linguistic terms is equal. It does not completely match the real problems. For instance, the DM may believe that the deviation between "fair" and "slight good" is less than the deviation between "slight good" and "good" in terms of the academic research level of the teacher. Moreover, the result of the operation may exceed the bounder limit of LTS. Thus, in order to respond flexibly and reasonably to this problem, in this section, by combining linguistic scale functions proposed by Wang et al. (2014), we innovate some novel operational laws for PFLS to make the results logical.

Definition 7 Let $S=\left\{s_{t} \mid t=0,1, \ldots, \tau-1\right\}$ be a LTS and $\lambda_{t}$ be a numeric value which represents the semantic of $s_{t}$. Then the linguistic scale functions (LSFs) $f$ is the mapping from $s_{t}$ to $\lambda_{t}(t=0,1 \ldots, \tau-1)$ is defined as:

$f: s_{t} \rightarrow \lambda_{t}(t=0,1, \ldots, \tau-1)$.

(1)The deviations between adjacent linguistic terms are equal, then

$f\left(s_{t}\right)=\lambda_{t}=\frac{t}{\tau-1}$.

(2)The deviations between adjacent linguistic terms are increasing with the extension from $s_{0}$, then

$$
\begin{aligned}
& f\left(s_{t}\right)=\lambda_{t} \\
& =\left\{\begin{array}{c}
\frac{\varsigma^{(\tau-1) / 2}-\varsigma^{(\tau-1-2 t) / 2}}{2 \varsigma^{(\tau-1) / 2}-2}(t=0,1, \ldots,(\tau-1) / 2) \\
\frac{\varsigma^{(\tau-1) / 2}+\varsigma^{(2 t-\tau+1) / 2}-2}{2 \varsigma^{(\tau-1) / 2}-2}(t=(\tau+1) / 2,(\tau+3) / 2, \ldots, \tau-1)
\end{array} .\right.
\end{aligned}
$$

where $\varsigma$ is a threshold, which can be determined according to the specific situations.

(3)The deviations between adjacent linguistic terms are decreasing with the extension from $s_{0}$, then

$f\left(s_{t}\right)=\lambda_{t}$ 


$$
=\left\{\begin{array}{l}
\frac{((\tau-1) / 2)^{a}-((\tau-1) / 2-t)^{a}}{2((\tau-1) / 2)^{a}}(t=0,1, \ldots,(\tau-1) / 2) \\
\frac{((\tau-1) / 2)^{b}+(t-(\tau-1) / 2)^{b}}{2((\tau-1) / 2)^{b}}(t=(\tau+1) / 2,(\tau+3) / 2, \ldots, \tau-1)
\end{array} .\right.
$$

where $a, b \in[0,1]$ can be determined based on the practical case. Especially, $a, b=1, f\left(s_{t}\right)=\frac{t}{\tau-1}$.

\subsection{Some novel operational laws for PFLS}

Considering that there are many deficiencies in the traditional PFLS algorithm, such as the operation result exceeds the upper bound of redefined LTS. In this part, we redefine some novel operational laws of PFLV based on linguistic scale functions, which are described as follows.

Definition 8 Let $H_{i}=\left\langle s_{\theta\left(x_{i}\right)},\left(P\left(x_{i}\right), I\left(x_{i}\right), N\left(x_{i}\right)\right)\right\rangle(i=$ $1,2,3)$ be any three PFLVs and $\lambda>0$. Then, based on LSFs, novel operational laws for PFLVs are given as follows:

(1) $H_{1} \oplus H_{2}=\left\langle f^{-1}\left(f\left(s_{\theta\left(x_{1}\right)}\right)+f\left(s_{\theta\left(x_{2}\right)}\right)-f\left(s_{\theta\left(x_{1}\right)}\right) f\right.\right.$ $\left.\left(s_{\theta\left(x_{2}\right)}\right)\right),\left(P\left(x_{1}\right)+P\left(x_{2}\right)-P\left(x_{1}\right) P\left(x_{2}\right), I\left(x_{1}\right) I\left(x_{2}\right)\right.$, $\left.\left.N\left(x_{1}\right) N\left(x_{2}\right)\right)\right\rangle$;

(2) $H_{1} \otimes H_{2}=\left\langle f^{-1}\left(f\left(s_{\theta\left(x_{1}\right)}\right) \times f\left(s_{\theta\left(x_{2}\right)}\right)\right),\left(P\left(x_{1}\right) P\left(x_{2}\right)\right.\right.$, $I\left(x_{1}\right)+I\left(x_{2}\right)-I\left(x_{1}\right) I\left(x_{2}\right), N\left(x_{1}\right)+N\left(x_{2}\right)-N\left(x_{1}\right)$ $\left.\left.N\left(x_{2}\right)\right)\right\rangle$;

(3) $\lambda H_{1}=\left\langle f^{-1}\left(1-\left(1-f\left(s_{\theta\left(x_{1}\right)}\right)\right)^{\lambda}\right),\left(1-\left(1-P\left(x_{1}\right)\right)^{\lambda}\right.\right.$, $\left.\left.I\left(x_{1}\right)^{\lambda}, N\left(x_{1}\right)^{\lambda}\right)\right\rangle$

(4) ${H_{1}}^{\lambda}=\left\langle f^{-1}\left(f\left(s_{\theta\left(x_{1}\right)}\right)^{\lambda}\right),\left(P\left(x_{1}\right)^{\lambda}, 1-\left(1-I\left(x_{1}\right)\right)^{\lambda}, 1-\right.\right.$ $\left.\left.\left(1-N\left(x_{1}\right)\right)^{\lambda}\right)\right\rangle$

(5) $H_{1}{ }^{C}=\left\langle f^{-1}\left(1-f\left(s_{\theta}\left(x_{1}\right)\right)\right),\left(N\left(x_{1}\right), I\left(x_{1}\right), P\left(x_{1}\right)\right)\right\rangle$.

Example 1 Continue to utilize the example mentioned earlier. $H_{1}=\left\langle s_{3},(0.5,0.2,0.1)\right\rangle$ and $H_{2}=\left\langle s_{5},(0.4,0.2,0.2)\right\rangle$ are two PFLVs. Suppose that $\mathrm{S}$ be a LTS with a granularity of 7, $f\left(s_{t}\right)$ is given as Eq.(6) and $\lambda=2$. By Definition 8, the following results can be obtained:

(1) $H_{1} \oplus H_{2}=\left\langle f^{-1}\left(f\left(s_{3}\right)+f\left(s_{5}\right)-f\left(s_{3}\right) f\left(s_{5}\right)\right),(0.5+\right.$ $0.4-0.5 \times 0.4,0.2 \times 0.2,0.1 \times 0.2)\rangle=\left\langle s_{5.5},(0.7,0.04\right.$, $0.02)\rangle$

(2) $H_{1} \oplus H_{2}=\left\langle f^{-1}\left(f\left(s_{3}\right) \times f\left(s_{5}\right)\right),(0.5 \times 0.4,0.2+0.2-\right.$ $0.2 \times 0.2,0.1+0.2-0.1 \times 0.2)\rangle=\left\langle s_{5.5},(0.2,0.36\right.$, $0.28)\rangle$

(3) $2 H_{1}=\left\langle f^{-1}\left(1-\left(1-f\left(s_{3}\right)\right)^{2}\right),\left(1-(1-0.5)^{2}, 0.2^{2}\right.\right.$, $\left.\left.0.1^{2}\right)\right\rangle=\left\langle s_{4.5},(0.75,0.04,0.01)\right\rangle$

(4) $\left(H_{1}\right)^{2}=\left\langle f^{-1}\left(f\left(s_{3}\right)\right)^{2}\right),\left((0.5)^{2}, 1-(1-0.2)^{2}, 1-\right.$ $\left.\left.(1-0.1)^{2}\right)\right\rangle=\left\langle s_{1.5},(0.25,0.36,0.19)\right\rangle$;

(5) $H_{1}{ }^{C}=\left\langle f^{-1}\left(1-f\left(s_{3}\right)\right),(0.1,0.2,0.5)\right\rangle=\left\langle s_{3},(0.1\right.$, $0.2,0.5)\rangle$.

Apparently, the above operational results are still PFLVs. Moreover, it can be found that the linguistic term in the calculation result does not exceed the upper limit of LTS, which illustrates the rationality and progress of the improved method we have proposed and ensures the credibility of the calculation result.

\subsection{The novel score and accuracy functions}

Definition 9 Suppose $S=\left\{s_{0}, s_{1}, \ldots, s_{\tau-1}\right\}$ be a LTS, $H=$ $\left\langle s_{\theta(x)},(P(x), I(x), N(x))\right\rangle$ be a PFLV. Then, the novel score function $M(H)$ of $H$ can be represented as:

$M(H)=\operatorname{Ind}\left(s_{\theta(H)}\right) \times\left(\frac{1+P(H)-N(H)}{2}\right)$.

the novel accuracy function $N F(H)$ of $H$ can be defined as:

$N F(H)=\operatorname{Ind}\left(s_{\theta(H)}\right) \times(P(H)+I(N)+N(H))$.

where $\operatorname{Ind}\left(s_{\theta(H)}\right)$ is the subscript of linguistic term $s_{\theta(H)}$, $P(H)$ is the $P(x)$ value of $H, I(H)$ is the $I(x)$ value of $H$ and $N(H)$ is the $N(x)$ value of $H$.

Definition 10 For any two PFLVs $H_{1}$ and $H_{2}$, then

(1) If $M\left(H_{1}\right)>M\left(H_{2}\right)$, then $H_{1} \succ H_{2}$;

(2) If $M\left(H_{1}\right)<M\left(H_{2}\right)$, then $H_{1} \prec H_{2}$;

(3) If $M\left(H_{1}\right)=M\left(H_{2}\right)$,then

(i) If $N F\left(H_{1}\right)>N F\left(H_{2}\right)$, then $H_{1} \succ H_{2}$;

(ii) If $N F\left(H_{1}\right)<N F\left(H_{2}\right)$, then $H_{1} \prec H_{2}$;

(iii) If $N F\left(H_{1}\right)=N F\left(H_{2}\right)$, then $H_{1} \sim H_{2}$;

Example 2 For $H_{1}=\left\langle s_{2}, 0.3,0.3,0.2\right\rangle$ and $H_{2}=\left\langle s_{2}, 0.4\right.$, $0.25,0.3\rangle$. If $f\left(s_{t}\right)$ is given as Eq.(6), $M\left(H_{1}\right)=M\left(H_{2}\right)=$ 0.1 . Then, the accuracy functions of the two PFLVs $N F\left(H_{1}\right)=$ 1.6 and $N F\left(H_{2}\right)=1.9$, therefore, $H_{2}>H_{1}$.

\section{Picture fuzzy linguistic Muirhead Mean aggregation operators}

In the light of the superiority of Muirhead Mean (MM) operators in coping with the interaction relationship between the input parameters, and taking into account attribute weights simultaneously. This section explains MM operators concerning PFLVs and suggests the MM aggregation operators with PFLVs along with Picture fuzzy linguistic weighted MM operator (PFLWMM) and Picture fuzzy linguistic weighted DMM operator (PFLWDMM).

\subsection{Picture fuzzy linguistic weighted MM operator}

Definition 11 Let $a_{j}(j=1,2, \ldots, n)$ be a group of PFLVs with their weight vector be $\omega_{i}=\left(\omega_{1}, \omega_{2}, \ldots, \omega_{n}\right)^{T}$, satisfy- 
ing $\omega_{i} \in[0,1]$ and $\sum_{i=1}^{n} \omega_{i}=1 . Q=\left(Q_{1}, Q_{2}, \ldots, Q_{n}\right) \in$ $R^{n}$ be a vector of parameters. Then the definition of the PFLWMM operator is expressed as:

$$
\begin{aligned}
& \operatorname{PFLWMM}_{n \omega}^{Q}\left(a_{1}, a_{2}, \ldots, a_{n}\right) \\
& =\left(\frac{1}{n !}\left(\underset{\sigma \in S_{n}}{\bigoplus}\left(\bigotimes_{j=1}^{n}\left(n \omega_{\sigma(j)} a_{\sigma(j)}\right) Q_{j}\right)\right)\right)^{\frac{1}{\sum_{j=1}^{n} Q_{j}}} .
\end{aligned}
$$

Theorem 1 Assume that $a_{j}(j=1,2, \cdots, n)$ be a set of PFLVs. The fused result obtained by PFLWMM operator is shown as

$$
\begin{aligned}
& \text { PFLWMM } M_{n \omega}^{Q}\left(a_{1}, a_{2}, \ldots, a_{n}\right) \\
& =\left(\frac{1}{n !}\left(\underset{\sigma \in S_{n}}{\oplus}\left(\bigotimes_{j=1}^{n}\left(n \omega_{\sigma(j)} a_{\sigma(j)}\right) Q_{j}\right)\right)\right)^{\frac{1}{\sum_{j=1}^{n} Q_{j}}} \\
& =\left\langlef ^ { - 1 } \left(\left( 1-\prod_{\sigma \in S_{n}}\left(1-\prod_{j=1}^{n}\right.\right.\right.\right. \\
& \left.\left.\left.\times\left(1-\left(1-f\left(s_{\theta\left(a_{\sigma(j)}\right)}\right)\right)^{n \omega_{\sigma(j)}}\right)^{Q_{j}}\right)^{\frac{1}{n !}}\right)^{\frac{1}{\sum_{j=1}^{n} Q_{j}}}\right), \\
& \left(1-\prod_{\sigma \in S_{n}}\right. \\
& \left.\times\left(1-\prod_{j=1}^{n}\left(1-\left(1-P\left(a_{\sigma(j)}\right)\right)^{n \omega_{\sigma(j)}}\right)^{Q_{j}}\right)^{\frac{1}{n !}}\right)^{\frac{1}{\sum_{j=1}^{n} Q_{j}}}, \\
& 1-\left(1-\prod_{\sigma \in S_{n}}\right. \\
& \left.\times\left(1-\prod_{j=1}^{n}\left(1-\left(1-I\left(a_{\sigma(j)}\right)\right)^{n \omega_{\sigma(j)}}\right)^{Q_{j}}\right)^{\frac{1}{n !}}\right)^{\frac{1}{\sum_{j=1}^{n} Q_{j}}}, \\
& 1-\left(1-\prod_{\sigma \in S_{n}}\left(1-\prod_{j=1}^{n}\right.\right. \\
& \left.\left.\left.\times\left(1-\left(1-N\left(a_{\sigma(j)}\right)\right)^{n \omega_{\sigma(j)}}\right)^{Q_{j}}\right)^{\frac{1}{n !}}\right)^{\frac{1}{\sum_{j=1}^{n} Q_{j}}}\right\rangle .
\end{aligned}
$$

where $n$ is the number of attributes, $\omega_{i}$ is weight vector.

\section{Proof (i) The linguistic set part:}

$$
\begin{aligned}
& n \omega_{\sigma(j)} s_{\theta\left(a_{\sigma(j)}\right)} \\
& \quad=f^{-1}\left(1-\left(1-f\left(s_{\theta\left(a_{\sigma(j)}\right)}\right)\right)^{n \omega_{\sigma(j)}}\right) .
\end{aligned}
$$

Then, $\underset{\sigma \in S_{n}}{\oplus}\left(\left(\omega_{\sigma(j)} s_{\theta\left(a_{\sigma(j)}\right)}\right)^{Q_{j}}\right)=\left\{f^{-1}\left(1-\prod_{\sigma \in S_{n}}\left(1-\prod_{j=1}^{n}\right.\right.\right.$ $\left.\left.\left.\left(1-\left(1-f\left(s_{\theta\left(a_{\sigma(j)}\right)}\right)\right)^{n \omega_{\sigma(j)}}\right) Q_{j}\right)\right)\right\}$

Thus,

$$
\left(\frac{1}{n !}\left(\underset{\sigma \in S_{n}}{\oplus}\left(\left(\omega_{\sigma(j)} s_{\theta\left(a_{\sigma(j)}\right)}\right)^{Q_{j}}\right)\right)\right)^{\frac{1}{\sum_{j=1}^{n} Q_{j}}}
$$

$$
\begin{aligned}
= & \left\{f ^ { - 1 } \left(\left(1-\prod_{\sigma \in S_{n}}\left(1-\prod_{j=1}^{n}(1-(1-f\right.\right.\right.\right. \\
& \left.\left.\left.\left.\left.\left.\times\left(s_{\theta\left(a_{\sigma(j)}\right)}\right)\right)^{\left.n \omega_{\sigma(j)}\right)}\right)_{j}\right)^{\frac{1}{n !}}\right)^{\frac{1}{\sum_{j=1}^{n} Q_{j}}}\right)\right\} .
\end{aligned}
$$

(ii)The Picture fuzzy set part:

$$
\begin{gathered}
\omega_{\sigma(j)} a_{\sigma(j)}=\left\{1-\left(1-P\left(a_{\sigma(j)}\right)\right)^{\omega_{\sigma(j)},},\right. \\
\left.\left(I\left(a_{\sigma(j)}\right)\right)^{\omega_{\sigma(j)}},\left(N\left(a_{\sigma(j)}\right)\right)^{\omega_{\sigma(j)}}\right\} .
\end{gathered}
$$

Then, $\underset{j=1}{\otimes}\left(\omega_{\sigma(j)} a_{\sigma(j)}\right)^{Q_{j}}=\left\{\prod_{j=1}^{n}(1-(1-P\right.$ $\left.\left(a_{\sigma(j)}\right)\right)^{\left.\omega_{\sigma(j)}\right)} Q_{j}, 1-\prod_{j=1}^{n}\left(1-\left(I\left(a_{\sigma(j)}\right)\right)^{\omega(j)}\right)^{Q_{j}}, 1-\prod_{j=1}^{n}$ $\left.\left(1-\left(N\left(a_{\sigma(j)}\right)\right)^{\omega(j)}\right)^{Q_{j}}\right\}$,

thus,

$$
\begin{aligned}
& \left(\frac{1}{n !}\left(\underset{\sigma \in S_{n}}{\oplus}\left(\stackrel{\otimes}{j=1}_{j}^{n}\left(\omega_{\sigma(j)} a_{\sigma(j)}\right)^{Q_{j}}\right)\right)\right)^{\frac{1}{\sum_{j=1}^{n} Q_{j}}} \\
& =\left\{\left(1-\prod_{\sigma \in S_{n}}\left(1-\prod_{j=1}^{n}\left(1-\left(1-P\left(a_{\sigma(j)}\right)\right)^{\omega_{\sigma(j)}}\right)^{Q_{j}}\right)^{\frac{1}{n !}}\right)^{\frac{1}{\sum_{j=1}^{n} Q_{j}}},\right. \\
& 1-\left(1-\prod_{\sigma \in S_{n}}\left(1-\prod_{j=1}^{n}\left(1-\left(1-I\left(a_{\sigma(j)}\right)\right)^{\omega_{\sigma(j)}}\right)^{Q_{j}}\right)^{\frac{1}{n !}}\right)^{\frac{1}{\sum_{j=1}^{n} Q_{j}}},
\end{aligned}
$$

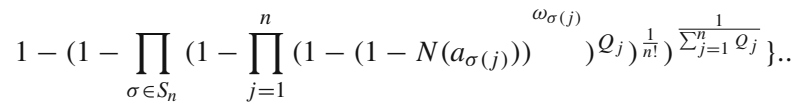

Therefore,

$$
\begin{aligned}
& \operatorname{PFLWMM} Q \underline{n \omega}\left(a_{1}, a_{2}, \ldots, a_{n}\right) \\
& =\left(\frac{1}{n !}\left(\underset{\sigma \in S_{n}}{\oplus}\left(\bigotimes_{j=1}^{n}\left(n \omega_{\sigma(j)} a_{\sigma(j)}\right)^{Q_{j}}\right)\right)\right)^{\frac{1}{\sum_{j=1}^{n} Q_{j}}} \\
& =\left\langlef ^ { - 1 } \left(\left( 1-\prod_{\sigma \in S_{n}}\left(1-\prod_{j=1}^{n}\right.\right.\right.\right. \\
& \left.\left.\times\left(1-\left(1-f\left(s_{\theta\left(a_{\sigma(j)}\right)}\right)\right)^{\left.n \omega_{\sigma(j)}\right)} Q^{Q_{j}}\right)^{\frac{1}{n !}}\right)^{\frac{1}{\sum_{j=1}^{n} Q_{j}}}\right), \\
& \left.\left(1-\prod_{\sigma \in S_{n}}\left(1-\prod_{j=1}^{n}\left(1-\left(1-P\left(a_{\sigma(j)}\right)\right)\right)^{n \omega_{\sigma(j)}}\right)^{Q_{j}}\right)^{\frac{1}{n !}}\right)^{\frac{1}{\sum_{j=1}^{n} Q_{j}}}, \\
& 1-\left(1-\prod_{\sigma \in S_{n}}\left(1-\prod_{j=1}^{n}\left(1-\left(1-I\left(a_{\sigma(j)}\right)\right)^{n \omega_{\sigma(j)}}\right)^{Q_{j}}\right)^{\frac{1}{n !}}\right)^{\frac{1}{\sum_{j=1}^{n} Q_{j}}}
\end{aligned}
$$

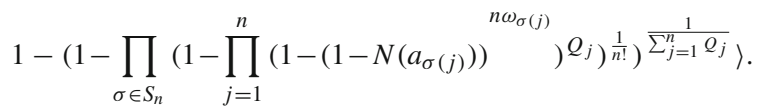

Hence, Eq.(12) is kept.

Example 3 Let $b_{1}=\left\langle s_{2}, 0.2,0.4,0.4\right\rangle, b_{2}=\left\langle s_{3}, 0.1,0.4\right.$, $0.5\rangle, b_{3}=\left\langle s_{2}, 0.3,0.5,0.2\right\rangle$ be three PFLVs. If $f\left(s_{t}\right)$ is 
given as Eq.(6), $Q=(0.2,0.3,0.5)$ and $\omega=(0.4,0.3,0.3)$, then according to Theorem 2, we can obtain:

$$
\begin{aligned}
& P F L W M M_{(0.4,0.3,0.3)}^{(0.2,0.3,0.5)}\left(\left\langle s_{2}, 0.2,0.4,0.4\right\rangle\right. \text {, } \\
& \left.\left\langle s_{3}, 0.1,0.4,0.5\right\rangle,\left\langle s_{2}, 0.3,0.5,0.2\right\rangle\right) \\
& =\left\langlef ^ { - 1 } \left(\left( 1-\left(\left(1-\left(1-\left(1-f\left(s_{2}\right)\right)^{1.2}\right)^{0.2}\right.\right.\right.\right.\right. \\
& \times\left(1-\left(1-f\left(s_{3}\right)\right)^{0.9}\right)^{0.3} \\
& \left.\times\left(1-\left(1-f\left(s_{2}\right)\right)^{0.9}\right)^{0.5}\right) \\
& \times\left(1-\left(1-\left(1-f\left(s_{2}\right)\right)^{1.2}\right)^{0.2} \times\left(1-\left(1-f\left(s_{2}\right)\right)^{0.9}\right)^{0.3}\right. \\
& \left.\times\left(1-\left(1-f\left(s_{3}\right)\right)^{0.9}\right)^{0.5}\right) \\
& \times\left(1-\left(1-\left(1-f\left(s_{3}\right)\right)^{0.9}\right)^{0.2} \times\left(1-\left(1-f\left(s_{2}\right)\right)^{1.2}\right)^{0.3}\right. \\
& \left.\times\left(1-\left(1-f\left(s_{2}\right)\right)^{0.9}\right)^{0.5}\right) \\
& \times\left(1-\left(1-\left(1-f\left(s_{3}\right)\right)^{0.9}\right)^{0.2} \times\left(1-\left(1-f\left(s_{2}\right)\right)^{0.9}\right)^{0.3}\right. \\
& \left.\times\left(1-\left(1-f\left(s_{2}\right)\right)^{1.2}\right)^{0.5}\right) \\
& \times\left(1-\left(1-\left(1-f\left(s_{2}\right)\right)^{0.9}\right)^{0.2} \times\left(1-\left(1-f\left(s_{2}\right)\right)^{1.2}\right)^{0.3}\right. \\
& \left.\times\left(1-\left(1-f\left(s_{3}\right)\right)^{0.9}\right)^{0.5}\right) \\
& \times\left(1-\left(1-\left(1-f\left(s_{2}\right)\right)^{0.9}\right)^{0.2} \times\left(1-\left(1-f\left(s_{3}\right)\right)^{0.9}\right)^{0.3}\right. \\
& \left.\left.\left.\left.\times\left(1-\left(1-f\left(s_{2}\right)\right)^{1.2}\right)^{0.5}\right)\right)^{\frac{1}{3 !}}\right)^{\frac{1}{1}}\right), \\
& 1-\left(\left(1-\left(1-(1-0.2)^{1.2}\right)^{0.2} \times\left(1-(1-0.1)^{0.9}\right)^{0.3}\right.\right. \\
& \left.\times\left(1-(1-0.3)^{0.9}\right)^{0.5}\right) \\
& \times\left(1-\left(1-(1-0.2)^{1.2}\right)^{0.2} \times\left(1-(1-0.3)^{0.9}\right)^{0.3}\right. \\
& \left.\times\left(1-(1-0.1)^{0.9}\right)^{0.5}\right) \\
& \times\left(1-\left(1-(1-0.1)^{0.9}\right)^{0.2} \times\left(1-(1-0.2)^{1.2}\right)^{0.3}\right. \\
& \left.\times\left(1-(1-0.3)^{0.9}\right)^{0.5}\right) \\
& \times\left(1-\left(1-(1-0.1)^{0.9}\right)^{0.2} \times\left(1-(1-0.3)^{0.9}\right)^{0.3}\right. \\
& \left.\times\left(1-(1-0.2)^{1.2}\right)^{0.5}\right) \\
& \times\left(1-\left(1-(1-0.3)^{0.9}\right)^{0.2} \times\left(1-(1-0.2)^{1.2}\right)^{0.3}\right. \\
& \left.\times\left(1-(1-0.1)^{0.9}\right)^{0.5}\right) \\
& \times\left(1-\left(1-(1-0.3)^{0.9}\right)^{0.2} \times\left(1-(1-0.1)^{0.9}\right)^{0.3}\right. \\
& \left.\left.\left.\times\left(1-(1-0.2)^{1.2}\right)^{0.5}\right)\right)^{\frac{1}{3 !}}\right)^{\frac{1}{1}} \\
& 1-\left(1-\left(\left(1-\left(1-0.4^{1.2}\right)^{0.2} \times\left(1-0.4^{0.9}\right)^{0.3}\right.\right.\right. \\
& \left.\times\left(1-0.5^{0.9}\right)^{0.5}\right) \\
& \times\left(1-\left(1-0.4^{1.2}\right)^{0.2} \times\left(1-0.5^{0.9}\right)^{0.3}\right. \\
& \left.\times\left(1-0.4^{0.9}\right)^{0.5}\right) \\
& \times\left(1-\left(1-0.4^{0.9}\right)^{0.2} \times\left(1-0.4^{1.2}\right)^{0.3}\right. \\
& \left.\times\left(1-0.5^{0.9}\right)^{0.5}\right) \\
& \times\left(1-\left(1-0.4^{0.9}\right)^{0.2} \times\left(1-0.5^{0.9}\right)^{0.3}\right. \\
& \left.\times\left(1-0.4^{1.2}\right)^{0.5}\right) \\
& \times\left(1-\left(1-0.5^{0.9}\right)^{0.2} \times\left(1-0.4^{1.2}\right)^{0.3}\right. \\
& \left.\times\left(1-0.4^{0.9}\right)^{0.5}\right) \\
& \times\left(1-\left(1-0.5^{0.9}\right)^{0.2} \times\left(1-0.4^{0.9}\right)^{0.3}\right. \\
& \left.\left.\left.\times\left(1-0.4^{1.2}\right)^{0.5}\right)\right)\right)^{\left.\frac{1}{3 !}\right)^{\frac{1}{1}},} \\
& 1-\left(1-\left(\left(1-\left(1-0.4^{1.2}\right)^{0.2} \times\left(1-0.5^{0.9}\right)^{0.3}\right.\right.\right. \\
& \left.\times\left(1-0.2^{0.9}\right)^{0.5}\right) \\
& \times\left(1-\left(1-0.4^{1.2}\right)^{0.2} \times\left(1-0.2^{0.9}\right)^{0.3}\right. \\
& \left.\times\left(1-0.5^{0.9}\right)^{0.5}\right) \\
& \times\left(1-\left(1-0.5^{0.9}\right)^{0.2} \times\left(1-0.4^{1.2}\right)^{0.3}\right.
\end{aligned}
$$

$$
\begin{aligned}
& \left.\times\left(1-0.2^{0.9}\right)^{0.5}\right) \\
& \times\left(1-\left(1-0.5^{0.9}\right)^{0.2} \times\left(1-0.2^{0.9}\right)^{0.3}\right. \\
& \left.\times\left(1-0.4^{1.2}\right)^{0.5}\right) \\
& \times\left(1-\left(1-0.2^{0.9}\right)^{0.2} \times\left(1-0.4^{1.2}\right)^{0.3}\right. \\
& \left.\times\left(1-0.5^{0.9}\right)^{0.5}\right) \\
& \times\left(1-\left(1-0.2^{0.9}\right)^{0.2} \times\left(1-0.5^{0.9}\right)^{0.3}\right. \\
& \left.\left.\left.\left.\left.\times\left(1-0.4^{1.2}\right)^{0.5}\right)\right)\right)^{\frac{1}{3 !}}\right)^{\frac{1}{1}}\right\rangle \\
& =\left\langle s_{2.268}, 0.208,0.441,0.344\right\rangle .
\end{aligned}
$$

Next, explore the desirable properties of the PFLWMM operator. Qualified operators should be able to give a clear order to different schemes in decision-making. This requires proof of its idempotency, boundedness and monotonicity, so as to avoid the situation where the operator gives the same sorting result as much as possible.

Property 1 (Idempotency). If $a_{j}=a=\left\langle s_{\theta(a)}, P(a), I(a)\right.$, $N(a)\rangle$ for all $j(j=1,2, \ldots, n)$, then

$P F L W M M_{n \omega}^{Q}\left(a_{1}, a_{2}, \ldots a_{n}\right)=a$.

Proof Since $a_{j}=a(j=1,2, \ldots, n)$, and $\sum_{i=1}^{n} \omega_{i}=1$, based on Theorem 2, we can get

$$
\begin{array}{rl}
P & F L W M M_{n \omega}^{Q}\left(a_{1}, a_{2}, \ldots, a_{n}\right) \\
= & \left(\frac{1}{n !}\left(\bigoplus_{\sigma \in S_{n}}\left(\bigoplus_{j=1}^{n}\left(n \omega_{\sigma(j)} a_{\sigma(j)}\right)^{Q_{j}}\right)\right)\right)^{\frac{1}{\sum_{j=1}^{n} Q_{j}}} \\
=\left\langle f^{-1}\left(\left(1-\prod_{\sigma \in S_{n}}\left(1-f\left(s_{\theta\left(a_{\sigma(j)}\right)}\right)^{\sum_{j=1}^{n} Q_{j}}\right)^{\frac{1}{n !}}\right)^{\frac{1}{\sum_{j=1}^{n} Q_{j}}}\right),\right. \\
& \left(\left(1-\prod_{\sigma \in S_{n}}\left(1-P\left(a_{\sigma(j)}\right)^{\sum_{j=1}^{n} Q_{j}}\right)^{\frac{1}{n !}}\right)^{\frac{1}{\sum_{j=1}^{n} Q_{j}}},\right. \\
& 1-\left(1-\prod_{\sigma \in S_{n}}\left(1-\left(1-I\left(a_{\sigma(j)}\right)\right)^{\sum_{j=1}^{n} Q_{j}}\right)^{\frac{1}{n !}}\right)^{\frac{1}{\sum_{j=1}^{n} Q_{j}}}, \\
& \left.1-\left(1-\prod_{\sigma \in S_{n}}\left(1-\left(1-N\left(a_{\sigma(j)}\right)^{\sum_{j=1}^{n} Q_{j}}\right)^{\frac{1}{n !}}\right)^{\frac{1}{\sum_{j=1}^{n} Q_{j}}}\right)\right\rangle \\
= & \left\langle f^{-1}\left(\left(f\left(s_{\theta\left(a_{\sigma(j)}\right)}\right)^{\sum_{j=1}^{n} Q_{j}}\right)^{\frac{1}{\sum_{j=1}^{n} Q_{j}}}\right)\right), \\
& \left(P\left(a_{\sigma(j)}\right)^{\sum_{j=1}^{n} Q_{j}}\right)^{\frac{1}{\sum_{j=1}^{n} Q_{j}}}, 1 \\
& -\left(\left(1-I\left(a_{\sigma(j)}\right)\right)^{\sum_{j=1}^{n} Q_{j}}\right)^{\sum_{j=1}^{n} Q_{j}}, \\
& \left.1-\left(\left(1-N\left(a_{\sigma(j)}\right)\right)^{\sum_{j=1}^{n} Q_{j}}\right)^{\overline{\sum_{j=1}^{n} Q_{j}}}\right\rangle=a .
\end{array}
$$

Then, the proof of Property 1 is completed.

Property 2 (Boundedness). Let $a_{j}=\left\langle s_{\theta\left(a_{j}\right)}, P\left(a_{j}\right), I\left(a_{j}\right)\right.$, $\left.N\left(a_{j}\right)\right\rangle(j=1,2, \ldots, n)$ be a lists of PFLVs. If $a_{j}^{-}=\min a_{j}$ 
and $a_{j}^{+}=\max a_{j}$, then

$a_{j}^{-} \leq P F L W M M_{n \omega}^{Q}\left(a_{1}, a_{2}, \ldots, a_{n}\right) \leq a_{j}^{+}$

Proof Since $a_{j}^{-} \leq a_{j} \leq a_{j}^{+}$for all $j(j=1,2, \ldots, n)$, let $a=\left\langle s_{\theta(a)}, P(a), I(a), N(a)\right\rangle$. It is obvious that

$\min \left\{s_{\theta\left(a_{j}\right)}\right\} \leq s_{\theta(a)} \leq \max \left\{s_{\theta\left(a_{j}\right)}\right\}, \min \left\{P\left(a_{j}\right)\right\} \leq$

$P(a) \leq \max \left\{P\left(a_{j}\right)\right\}$

$\min \left\{I\left(a_{j}\right)\right\} \leq I(a) \leq \max \left\{I\left(a_{j}\right)\right\}, \min \left\{N\left(a_{j}\right)\right\} \leq$ $N(a) \leq \max \left\{N\left(a_{j}\right)\right\}$

According to properties 1-2, $a_{j}^{-} \leq \operatorname{PFLWMM} \underset{n \omega}{Q}\left(a_{1}\right.$, $\left.a_{2}, \ldots, a_{n}\right) \leq a_{j}^{+}$. Therefore, the proof of Property 2 is completed.

Property 3 (Monotonicity). Assume that $a_{j}=\left\langle s_{\theta\left(a_{j}\right)}\right.$, $\left.P\left(a_{j}\right), I\left(a_{j}\right), N\left(a_{j}\right)\right\rangle$ and $b_{j}=\left\langle s_{\theta\left(b_{j}\right)}, P\left(b_{j}\right), I\left(b_{j}\right)\right.$, $\left.N\left(b_{j}\right)\right\rangle(j=1,2, \ldots, n)$ are two lists of PFLVs. Let $a_{j} \leq b_{j}$ for all $j$, then

$$
\begin{aligned}
& P F L W M M_{n \omega}^{Q}\left(a_{1}, a_{2}, \ldots, a_{n}\right) \\
& \quad \leq P F L W M M_{n \omega}^{Q}\left(b_{1}, b_{2}, \ldots, b_{n}\right) .
\end{aligned}
$$

Proof Since $a_{j} \leq b_{j}$ for all $j$, we can get

$$
\begin{aligned}
& \prod_{j=1}^{n}\left(1-\left(1-P\left(a_{\sigma(j)}\right)\right)^{\omega_{\sigma(j)}}\right)^{Q_{J}} \\
& \quad \leq \prod_{j=1}^{n}\left(1-\left(1-P\left(b_{\sigma(j)}\right)\right)^{\omega_{\sigma(j)}}\right)^{Q_{J}} .
\end{aligned}
$$

Thus,

$$
\begin{aligned}
& \left(1-\prod_{\sigma \in S_{n}}\left(1-\prod_{j=1}^{n}\left(1-\left(1-P\left(a_{\sigma(j)}\right)\right)^{\omega_{\sigma(j)}}\right)^{Q_{J}}\right)^{\frac{1}{n !}}\right)^{\frac{1}{\sum_{j=1}^{n} Q_{j}}} \\
& \leq\left(1-\prod_{\sigma \in S_{n}}\left(1-\prod_{j=1}^{n}\left(1-\left(1-P\left(b_{\sigma(j)}\right)\right)^{\omega_{\sigma(j)}}\right)^{Q_{J}}\right)^{\left.\frac{1}{n !}\right)^{\frac{1}{\sum_{j=1}^{n} Q_{j}}} .}\right.
\end{aligned}
$$

That's $P(a) \leq P(b)$. Similarity, we can obtain $s_{\theta(a)} \leq$ $s_{\theta(b)}, I(a) \leq I(b)$ and $N(a) \geq N(b)$, therefore, $P F \bar{L}$ $W M M_{n \omega}^{Q}\left(a_{1}, a_{2}, \ldots, a_{n}\right) \leq P F L W M M_{n \omega}^{Q}\left(b_{1}, b_{2}, \ldots, b_{n}\right)$. So, the Property 3 is right.

PFLWMM operator does not have the property of Commutatively.

Remark 1 In the case where $\omega=(1 / n, 1 / n, \ldots, 1 / n)^{T}$, then PFLWMM operator degenerates into the PFLMM operator.

$$
\begin{aligned}
& \operatorname{PFLMM} M^{Q}\left(a_{1}, a_{2}, \ldots, a_{n}\right) \\
& \quad=\left\langle f^{-1}\left(\left(1-\prod_{\sigma \in S_{n}}\left(1-\prod_{j=1}^{n} f\left(s_{\theta\left(a_{\sigma(j)}\right)}\right)^{Q_{j}}\right)^{\frac{1}{n !}}\right)^{\frac{1}{\sum_{j=1}^{n} Q_{j}}}\right),\right.
\end{aligned}
$$

$$
\begin{aligned}
& \left(1-\prod_{\sigma \in S_{n}}\left(1-\prod_{j=1}^{n} P\left(a_{\sigma(j)}\right)^{Q_{j}}\right)^{\frac{1}{n !}}\right)^{\frac{1}{\sum_{j=1}^{n} Q_{j}}}, \\
& 1-\left(1-\prod_{\sigma \in S_{n}}\left(1-\prod_{j=1}^{n}\left(1-I\left(a_{\sigma(j)}\right)\right)^{Q_{j}}\right)^{\frac{1}{n !}}\right)^{\frac{1}{\sum_{j=1}^{n} Q_{j}}}, \\
& 1-\left(1-\prod_{\sigma \in S_{n}}\left(1-\prod_{j=1}^{n}\left(1-N\left(a_{\sigma(j)}\right)\right)^{\frac{1}{n !}}\right)^{\frac{1}{\sum_{j=1}^{n} Q_{j}}}\right\rangle
\end{aligned}
$$

\subsection{Picture fuzzy linguistic weighted DMM operator}

Definition 12 Let $a_{j}(j=1,2, \ldots, n)$ be a group of PFLVs with their weight vector be $\omega_{i}=\left(\omega_{1}, \omega_{2}, \ldots, \omega_{n}\right)^{T}$, satisfying $\omega_{i} \in[0,1]$ and $\sum_{i=1}^{n} \omega_{i}=1 . Q=\left(Q_{1}, Q_{2}, \ldots, Q_{n}\right) \in$ $R^{n}$ be a vector of parameters. Then the definition of the PFLWDMM operator is expressed as:

$$
\begin{aligned}
& P F L W D M M_{n \omega}^{Q}\left(a_{1}, a_{2}, \ldots, a_{n}\right) \\
& =\frac{1}{\sum_{j=1}^{n} Q_{j}}\left(\underset{\sigma \in S_{n}}{\otimes}\left(\bigoplus_{j=1}^{n}\left(Q_{j} a_{\sigma(j)}^{n \omega_{\sigma(j)}}\right)\right)\right)^{\frac{1}{n !}} .
\end{aligned}
$$

Theorem 2 Assume that $a_{j}(j=1,2, \cdots, n)$ be a set of PFLVs. The fused values obtained by PFLWDMM operator are shown as:

$$
\begin{aligned}
& P F L W D M M_{n \omega}^{Q}\left(a_{1}, a_{2}, \ldots, a_{n}\right) \\
& =\frac{1}{\sum_{j=1}^{n} Q_{j}}\left(\underset{\sigma \in S_{n}}{\otimes}\left(\stackrel{\oplus=1}{n}_{j=1}\left(Q_{j} a_{\sigma(j)}^{n \omega_{\sigma(j)}}\right)\right)\right)^{\frac{1}{n !}} \\
& =\left\langlef ^ { - 1 } \left(\left( 1-\prod_{\sigma \in S_{n}}\left(1-\prod_{j=1}^{n}\right.\right.\right.\right. \\
& \left.\left.\left.\times\left(1-\left(1-f\left(s_{\theta\left(a_{\sigma(j)}\right)}\right)\right)^{n \omega_{\sigma(j)}}\right)^{Q_{j}}\right)^{\frac{1}{n !}}\right)^{\sum_{j=1}^{n} Q_{j}}\right),
\end{aligned}
$$

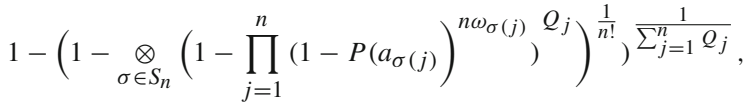

$$
\begin{aligned}
& \left(1-\prod_{\sigma \in S_{n}}\left(1-\prod_{j=1}^{n}\left(1-\left(1-I\left(a_{\sigma(j)}\right)\right)^{n \omega_{\sigma(j)}}\right)^{Q_{j}}\right)^{\left.\frac{1}{n !}\right)^{\sum_{j=1}^{n} Q_{j}}}\right.
\end{aligned}
$$

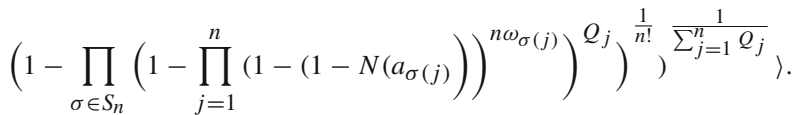

The proof process is similar to the PFLWMM operator, its omitted the proof.

Example 4 Let $d_{1}=\left\langle s_{2}, 0.2,0.4,0.4\right\rangle, d_{2}=\left\langle s_{3}, 0.1,0.4\right.$, $0.5\rangle, d_{3}=\left\langle s_{2}, 0.3,0.5,0.2\right\rangle$ be three PFLVs. If $f\left(s_{t}\right)$ is 
given as Eq.(6), $Q=(0.2,0.3,0.5)$ and $\omega=(0.4,0.3,0.3)$, then according to Theorem 3, we can obtain:

$$
\begin{aligned}
& \text { P F LW DMM M } M_{(0.4,0.4,0.2)}^{(0.2,0.3,0.5)}\left(\left\langle s_{1}, 0.3,0.2,0.4\right\rangle,\left\langle s_{3}, 0.1,0.4\right. \text {, }\right. \\
& \left.0.5\rangle,\left\langle s_{2}, 0.4,0.3,0.3\right\rangle\right) \\
& =\left\langlef ^ { - 1 } \left(\left( 1-\left(1-\left(\left(1-\left(1-f\left(s_{1}\right)^{1.2}\right)^{0.2}\right.\right.\right.\right.\right.\right. \\
& \left.\times\left(1-f\left(s_{3}\right)^{1.2}\right)^{0.3}\left(1-f\left(s_{2}\right)^{0.6}\right)^{0.5}\right) \\
& \times\left(1-\left(1-f\left(s_{1}\right)^{1.2}\right)^{0.2} \times\left(1-f\left(s_{2}\right)^{0.6}\right)^{0.3}\right. \\
& \left.\times\left(1-f\left(s_{3}\right)^{1.2}\right)^{0.5}\right) \\
& \times\left(1-\left(1-f\left(s_{3}\right)^{1.2}\right)^{0.2} \times\left(1-f\left(s_{1}\right)^{1.2}\right)^{0.3}\right. \\
& \left.\times\left(1-f\left(s_{2}\right)^{0.6}\right)^{0.5}\right) \\
& \times\left(1-\left(1-f\left(s_{3}\right)^{1.2}\right)^{0.2} \times\left(1-f\left(s_{2}\right)^{0.6}\right)^{0.3}\right. \\
& \left.\times\left(1-f\left(s_{1}\right)^{1.2}\right)^{0.5}\right) \\
& \times\left(1-\left(1-f\left(s_{2}\right)^{0.6}\right)^{0.2} \times\left(1-f\left(s_{1}\right)^{1.2}\right)^{0.3}\right. \\
& \left.\times\left(1-f\left(s_{3}\right)^{1.2}\right)^{0.5}\right) \\
& \times\left(1-\left(1-f\left(s_{2}\right)^{0.6}\right)^{0.2} \times\left(1-f\left(s_{3}\right)^{1.2}\right)^{0.3}\right. \\
& \left.\left.\left.\left.\times\left(1-f\left(s_{1}\right)^{1.2}\right)^{0.5}\right)\right)^{\frac{1}{3 !}}\right)\right) \text {, } \\
& \left(1-\left(1-\left(\left(1-\left(1-0.3^{1.2}\right)^{0.2} \times\left(1-0.1^{1.2}\right)^{0.3}\right.\right.\right.\right. \\
& \left.\times\left(1-0.4^{0.6}\right)^{0.5}\right) \\
& \times\left(1-\left(1-0.3^{1.2}\right)^{0.2} \times\left(1-0.4^{0.6}\right)^{0.3}\right. \\
& \left.\times\left(1-0.1^{1.2}\right)^{0.5}\right) \\
& \times\left(1-\left(1-0.1^{1.2}\right)^{0.2} \times\left(1-0.3^{1.2}\right)^{0.3}\right. \\
& \left.\times\left(1-0.4^{0.6}\right)^{0.5}\right) \\
& \times\left(1-\left(1-0.4^{0.6}\right)^{0.2} \times\left(1-0.3^{1.2}\right)^{0.3}\right. \\
& \left.\times\left(1-0.1^{1.2}\right)^{0.5}\right) \\
& \times\left(1-\left(1-0.4^{0.6}\right)^{0.2} \times\left(1-0.1^{1.2}\right)^{0.3}\right. \\
& \left.\times\left(1-0.3^{1.2}\right)^{0.5}\right) \\
& \times\left(1-\left(1-0.3^{1.2}\right)^{0.2} \times\left(1-0.1^{1.2}\right)^{0.3}\right. \\
& \left.\left.\left.\times\left(1-0.4^{0.6}\right)^{0.5}\right)\right)^{\frac{1}{3 !}}\right)^{\frac{1}{1}} \text {, } \\
& \left(1-\left(\left(1-\left(1-(1-0.2)^{1.2}\right)^{0.2} \times\left(1-(1-0.4)^{1.2}\right)^{0.3}\right.\right.\right. \\
& \left.\times\left(1-(1-0.3)^{0.6}\right)^{0.5}\right) \\
& \times\left(1-\left(1-(1-0.2)^{1.2}\right)^{0.2} \times\left(1-(1-0.3)^{0.6}\right)^{0.3}\right. \\
& \left.\times\left(1-(1-0.4)^{1.2}\right)^{0.5}\right) \\
& \times\left(1-\left(1-(1-0.4)^{1.2}\right)^{0.2} \times\left(1-(1-0.2)^{1.2}\right)^{0.3}\right. \\
& \left.\times\left(1-(1-0.3)^{0.6}\right)^{0.5}\right) \\
& \times\left(1-\left(1-(1-0.4)^{1.2}\right)^{0.2} \times\left(1-(1-0.3)^{0.6}\right)^{0.3}\right. \\
& \left.\times\left(1-(1-0.2)^{1.2}\right)^{0.5}\right) \\
& \times\left(1-\left(1-(1-0.3)^{0.6}\right)^{0.2} \times\left(1-(1-0.4)^{1.2}\right)^{0.3}\right. \\
& \left.\times\left(1-(1-0.2)^{1.2}\right)^{0.5}\right) \\
& \times\left(1-\left(1-(1-0.3)^{0.6}\right)^{0.2} \times\left(1-(1-0.2)^{1.2}\right)^{0.3}\right. \\
& \left.\left.\left.\times\left(1-(1-0.4)^{1.2}\right)^{0.5}\right)\right)^{\frac{1}{3 !}}\right)^{\frac{1}{1}}, \\
& \left(1-\left(\left(1-\left(1-(1-0.4)^{1.2}\right)^{0.2} \times\left(1-(1-0.5)^{1.2}\right)^{0.3}\right.\right.\right. \\
& \left.\times\left(1-(1-0.3)^{0.6}\right)^{0.5}\right) \\
& \times\left(1-\left(1-(1-0.4)^{1.2}\right)^{0.2} \times\left(1-(1-0.3)^{0.6}\right)^{0.3}\right. \\
& \left.\times\left(1-(1-0.5)^{1.2}\right)^{0.5}\right) \\
& \times\left(1-\left(1-(1-0.5)^{1.2}\right)^{0.2} \times\left(1-(1-0.4)^{1.2}\right)^{0.3}\right. \\
& \left.\times\left(1-(1-0.3)^{0.6}\right)^{0.5}\right) \\
& \times\left(1-\left(1-(1-0.5)^{1.2}\right)^{0.2} \times\left(1-(1-0.3)^{0.6}\right)^{0.3}\right.
\end{aligned}
$$

$$
\begin{aligned}
& \left.\times\left(1-(1-0.4)^{1.2}\right)^{0.5}\right) \\
& \times\left(1-\left(1-(1-0.3)^{0.6}\right)^{0.2} \times\left(1-(1-0.5)^{1.2}\right)^{0.3}\right. \\
& \left.\times\left(1-(1-0.4)^{1.2}\right)^{0.5}\right) \\
& \times\left(1-\left(1-(1-0.3)^{0.6}\right)^{0.2} \times\left(1-(1-0.4)^{1.2}\right)^{0.3}\right. \\
& \left.\left.\left.\left.\left.\times\left(1-(1-0.5)^{1.2}\right)^{0.5}\right)\right)^{\frac{1}{3 !}}\right)^{\frac{1}{1}}\right)\right\rangle=\left\langle s_{2.232}, 0.320,0.277,0.372\right\rangle
\end{aligned}
$$

Similarly, in order to further illustrate that the PFLWDMM operator can give a clear order to different schemes and can avoid the same ordering situation as much as possible, the three properties that the operator satisfies are expressed as follows.

Property 4 (Idempotency). If all $a_{j}(j=1,2, \ldots, n)$ are equal, i.e., $a_{j}=a$ for all $j$, then

$P F L W D M M_{n \omega}^{Q}\left(a_{1}, a_{2}, \ldots, a_{n}\right)=a$.

Property 5 (Boundedness). Let $a_{j}=\left\langle s_{\theta\left(a_{j}\right)}, P\left(a_{j}\right), I\left(a_{j}\right)\right.$, $\left.N\left(a_{j}\right)\right\rangle(j=1,2, \ldots, n)$ be a lists of PFLVs. If $a_{j}^{-}=\min a_{j}$ and $a_{j}^{+}=\max a_{j}$, then

$a_{j}^{-} \leq P F L W D M M_{n \omega}^{Q}\left(a_{1}, a_{2}, \ldots, a_{n}\right) \leq a_{j}^{+}$.

Property 6 (Monotonicity). Assume that $a_{j}=\left\langle s_{\theta\left(a_{j}\right)}\right.$, $\left.P\left(a_{j}\right), I\left(a_{j}\right), N\left(a_{j}\right)\right\rangle$ and $b_{j}=\left\langle s_{\theta\left(b_{j}\right)}, P\left(b_{j}\right), I\left(b_{j}\right), N\left(b_{j}\right)\right\rangle$ are two lists of PFLVs. Let $a_{j} \leq b_{j}$ for all $j$, then

$P F L W D M M_{n \omega}^{Q}\left(a_{1}, a_{2}, \ldots, a_{n}\right) \leq P F L W D M M_{n \omega}^{Q}\left(b_{1}, b_{2}, \ldots, b_{n}\right)$.

The proof of above three properties is similar to PFLWMM, so the proof is omitted.

Remark 2 In the case where $\omega=(1 / n, 1 / n, \ldots, 1 / n)^{T}$, then PFLWDMM operator degenerate into the PFLDMM operator.

$$
\begin{aligned}
& \operatorname{PFLDMM}^{Q}\left(a_{1}, a_{2}, \ldots, a_{n}\right) \\
& =\left\langlef ^ { - 1 } \left( 1-\left(1-\underset{\sigma \in S_{n}}{\otimes}\left(1-\prod_{j=1}^{n}\right.\right.\right.\right. \\
& \left.\left.\left.\left(1-s_{\theta\left(a_{\sigma(j)}\right)}\right)^{Q_{j}}\right)^{\frac{1}{n !}}\right)^{\frac{1}{\sum_{j=1}^{n} Q_{j}}}\right), \\
& 1-\left(1-\otimes_{\sigma \in S_{n}}\left(1-\prod_{j=1}^{n}\left(1-P\left(a_{\sigma(j)}\right)\right)^{Q_{j}}\right)^{\frac{1}{n !}}\right)^{\frac{1}{\sum_{j=1}^{n} Q_{j}}}, \\
& \left(1-\prod_{\sigma \in S_{n}}\left(1-\prod_{j=1}^{n}\left(I\left(a_{\sigma(j)}\right)\right)^{Q_{j}}\right)^{\frac{1}{n !}}\right)^{\frac{1}{\sum_{j=1}^{n} Q_{j}}}, \\
& \left(1-\prod_{\sigma \in S_{n}}\left(1-\prod_{j=1}^{n}\left(N\left(a_{\sigma(j)}\right)\right)^{Q_{j}}\right)^{\frac{1}{n !}}\right)^{\left.\frac{1}{\sum_{j=1}^{n} Q_{j}}\right\rangle} .
\end{aligned}
$$




\section{Multiple attribute decision-making algorithm based on picture fuzzy linguistic information}

In this part, we shall develop a new algorithm based on the Picture fuzzy linguistic aggregation operators to solve MADM problems under picture fuzzy linguistic environment. Let $A=\left\{A_{1}, A_{2}, \ldots, A_{m}\right\}$ be the set of alternatives, $C=\left\{C_{1}, C_{2}, \ldots, C_{n}\right\}$ be the set of attributes, and $\omega=$ $\left\{\omega_{1}, \omega_{2}, \ldots, \omega_{n}\right\}$ be the weight of attribute such that $\omega_{j}>$ $0(j=1,2, \ldots, n)$ and $\sum_{i=1}^{n} \omega_{j}=1$. Suppose that $H=$ $\left(h_{i j}\right)_{m \times n}=\left\langle s_{i j},\left(P_{i j}, I_{i j}, N_{i j}\right)\right\rangle_{m \times n}$ is the picture fuzzy linguistic decision matrix, where $h_{i j}$ is an PFLV and expresses the evaluation value of alternative $A_{i}$ with respect to the attribute $C_{j}$ by the decision maker.

In the following, we utilize the PFLWMM (PFLWDMM) operator to solve MADM problems with PFLV information. The following steps are provided to find the best alternatives. The flowchart of the proposed method is shown in Fig. 1.

Step 1. Normalize the attribute values. In a real problem, there are two types of attributes, then we need transformed cost type into benefit one to construct a standardized decision-making matrices by utilizing Eq.(17). (For convenience, standardized decision-making matrices still expressed by $\left.H=\left(h_{i j}\right)_{m \times n}\right)$

$h_{i j}=\left\langle f^{-1}\left(1-f\left(s_{h_{i j}}\right)\right), N\left(h_{i j}\right), I\left(h_{i j}\right), P\left(h_{i j}\right)\right\rangle$.

Step 2. Aggregate all attribute values $h_{i j}(j=1,2, \ldots, n)$ of each alternative to the comprehensive values $h_{i}$ by utilizing the PFLWMM operator defined by Definition 11 or PFLWDMM operator defined by Definition 12 .

Step 3. Compute the score values $E\left(h_{i}\right)(i=1,2, \ldots, m)$ of the PFLVs $h_{i}$ by Eq.(9). If the values of score function are same, we will use the accuracy function to further comparison.

Step 4. Order all the alternatives $A_{i}$ by the comparison method of PFLVs and select the best choice by $E\left(h_{i}\right)$ or $H\left(h_{i}\right)$.

\section{Step 5. End.}

\section{Illustrative example and comparative analysis}

In this section, we shall present a numerical example concerning the selection of the innovative "Mobike" sharing bike design, which is adapted from Liao et al. (2018) to show the feasibility and applicability of the proposed models.

\section{1 background}

In order to solve the "last few kilometers of travel" problem, sharing bike as a new form of sharing economy appear in people's field of vision. Because of no limit of time and place for taking and parking bikes, and attracted more and more people attentions. "Mobike" is one of many shared cycling brands. In order to enhance the competitiveness of its brand, "Mobike" pays much more attention at the beginning of the bicycle design phase to make the designed bicycle more comfortable and safe.

Assume that "Mobike" company want to select the optimal bicycle design from four bicycle production factory $\left\{A_{1}, A_{2}, A_{3}, A_{4}\right\}$, after thoughtful survey of the information, the "Mobike" company have considered the following key criteria, including comfort $C_{1}$, practicality $C_{2}$, versatility $C_{3}$ and security of $C_{4}$, whose weighted vector is $\omega=(0.25,0.3,0.25,0.2)^{T}$. The linguistic term set $S=$ $\left\{s_{0}, s_{1}, \ldots, s_{6}\right\}$ is utilized. The specific semantic of the linguistic term set is expressed as: $s_{0}=$ extremely poor, $s_{1}$ $=$ poor, $s_{2}=$ slight poor, $s_{3}=$ fair, $s_{4}=$ slight good, $s_{5}=$ good, $s_{6}=$ extremely good. For example, twelve experts are invited to assess the optimal bike design, $s_{4}$ is the linguistic variable, which is an evaluation value for alternative $A_{1}$ with respect to attribute $C_{1}$. On the day of the judging, only eleven experts participate in the assessment, and one person was absent. Suppose that four out of eleven experts voted for $s_{4}$, six remained neutral, and one people voted against, then evaluation information given by experts can be denoted by $<s_{4}, 4 / 12,6 / 12,1 / 12>$. Then, the decision matrixes is shown in Table 1.

Then, we can use the models developed in Sect. 5 for obtaining the optimal alternative. The following steps are involved:

Step 1. Normalize attribute values. In this example, because of all attributes are the benefit type, so there is no need for transformation.

Step 2. Based on Table 1, aggregate the attribute values $h_{i j}(j=1,2, \ldots, n)$ of each alternative by utilizing the PFLWMM operator given in Eq.(12) or PFLWDMM operator given in Eq.(15) to obtain the overall values $h_{i}(i=1,2, \ldots, m)$. If $f\left(s_{t}\right)$ is given as Eq.(6) and $Q=$ $(0.2,0.3,0.2,0.3)$, then, the fused results are listed in Table 2.

Step 3. Compute the score values of each alternative combined with Eq.(9), the results are shown in Table 3.

Step 4. Based on the score values of the overall alternative present in Table 3 . We can rank all the alternatives by utilizing Definition 10. It is obviously find that the ranking of the bike production factory based on the different aggregation 


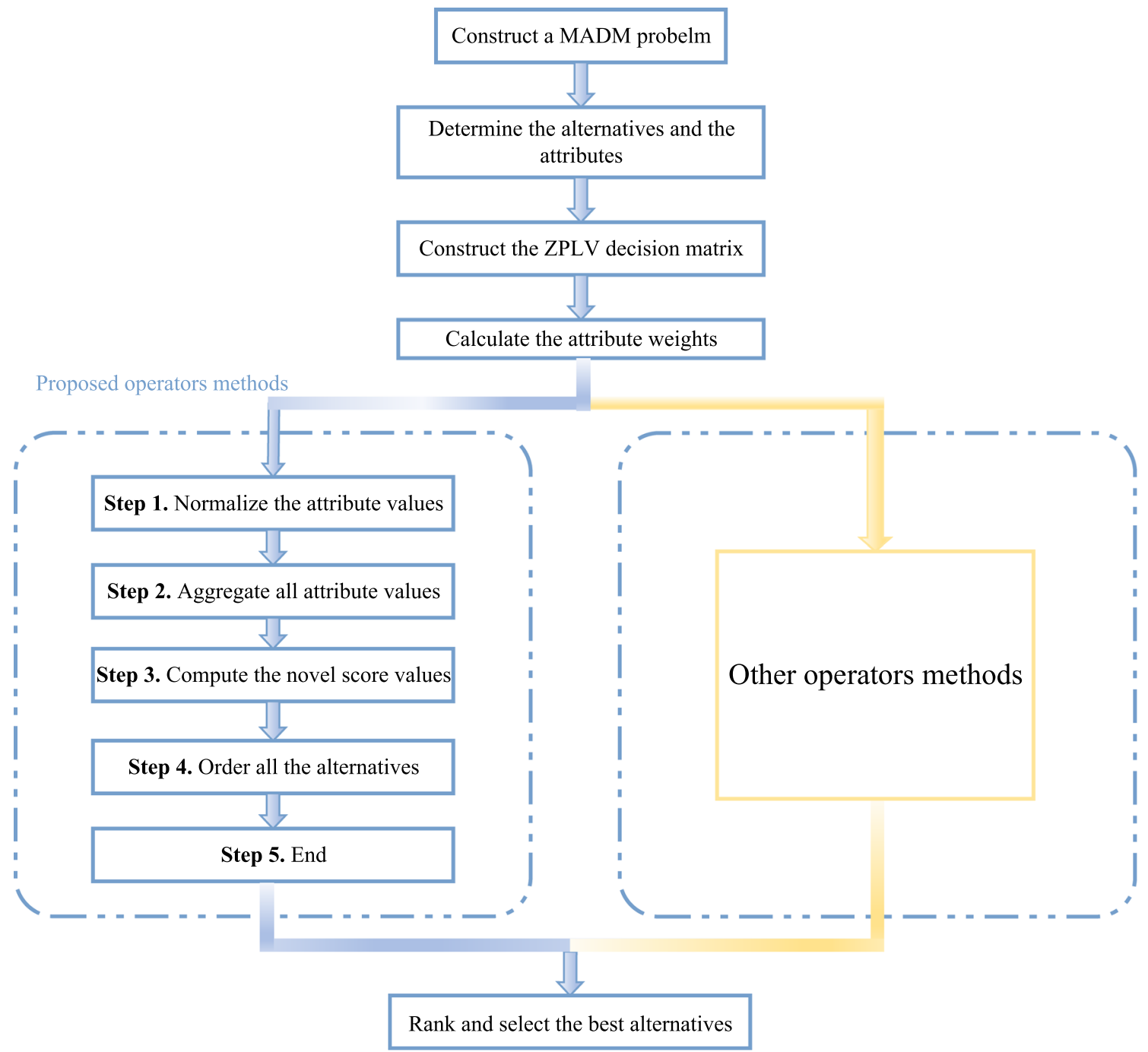

Fig. 1 The proposed algorithm based on the PFLV aggregation operators to solve MADM problems

Table 1 The Picture fuzzy linguistic information decision matrix

\begin{tabular}{cllll}
\hline Alternative & $\tilde{C}_{1}$ & $\tilde{C}_{2}$ & $\tilde{C}_{3}$ & $\tilde{C}_{4}$ \\
\hline$A_{1}$ & $<s_{4}, 4 / 12,6 / 12,1 / 12>$ & $<s_{2}, 6 / 12,4 / 12,2 / 12>$ & $<s_{3}, 4 / 12,7 / 12,1 / 12>$ & $<s_{4}, 2 / 12,9 / 12,1 / 12>$ \\
$A_{2}$ & $<s_{4}, 2 / 12,7 / 12,2 / 12>$ & $<s_{3}, 6 / 12,3 / 12,2 / 12>$ & $<s_{2}, 5 / 12,4 / 12,3 / 12>$ & $<s_{5}, 8 / 12,2 / 12,1 / 12>$ \\
$A_{3}$ & $<s_{2}, 10 / 12,1 / 12,1 / 12>$ & $<s_{1}, 8 / 12,1 / 12,3 / 12>$ & $<s_{5}, 2 / 12,8 / 12,1 / 12>$ & $<s_{3}, 2 / 12,8 / 12,2 / 12>$ \\
$A_{4}$ & $<s_{3}, 2 / 12,8 / 12,1 / 12>$ & $<s_{4}, 9 / 12,2 / 12,1 / 12>$ & $<s_{4}, 7 / 12,4 / 12,1 / 12>$ & $<s_{3}, 2 / 12,8 / 12,1 / 12>$ \\
\hline
\end{tabular}

Table 2 The aggregating results by the PFLWMM (PFLWDMM) operator

\begin{tabular}{cll}
\hline Alternative & $\tilde{P} F L W M M$ & $\tilde{P} F L W D M M$ \\
\hline$A_{1}$ & $<s_{3.144}, 0.249,0.619,0.098>$ & $<s_{3.426}, 0.301,0.547,0.09>$ \\
$A_{2}$ & $<s_{3.342}, 0.362,0.347,0.174>$ & $<s_{3.822}, 0.443,0.296,0.160>$ \\
$A_{3}$ & $<s_{2.352}, 0.247,0.602,0.096>$ & $<s_{3.276}, 0.586,0.232,0.068>$ \\
$A_{4}$ & $<s_{3.606}, 0.355,0.488,0.074>$ & $<s_{3.942}, 0.621,0.234,0.069>$ \\
\hline
\end{tabular}


Table 3 The score function of the different sharing bicycle design

\begin{tabular}{cll}
\hline Alternative & \multicolumn{1}{c}{$\tilde{P} F W M M$} & $\tilde{P} F L W D M M$ \\
\hline$A_{1}$ & 1.809 & 2.074 \\
$A_{2}$ & 1.985 & 2.452 \\
$A_{3}$ & 1.354 & 2.486 \\
$A_{4}$ & 2.310 & 3.059 \\
\hline
\end{tabular}

Table 4 Ranking of the different sharing bicycle design

\begin{tabular}{ll}
\hline Alternative & Ordering \\
\hline PFLWMM & $A_{4} \succ A_{2} \succ A_{1} \succ A_{3}$ \\
$P F L W D M M$ & $A_{4} \succ A_{3} \succ A_{2} \succ A_{1}$ \\
\hline
\end{tabular}

operator is slightly different. The order results are shown in Table 4.

Step 5. The bicycle production factory $A_{4}$ is the optimal choose.

\subsection{Comparative analysis}

For proving the prominent advantages of the proposed methods under picture fuzzy linguistic environment, we compare the proposed methods with existing methods such as the methods based on PFLNWAA Ashraf et al. (2018), PFLNWGA Ashraf et al. (2018) and A-PFLWAA Liu and Zhang (2018) to rank this example and the ranking results are presented in Table 5 and Fig. 1.

We can clearly find that the result of the best alternative obtained by our proposed operators is consistent with other exiting operators and represent $A_{4}$ is the best solution. This effectively proves the validity and rationality of our proposed method. Further, it is clear that the overall ranking order based on the PFLNWGA and A-PFLWAA operators are identical although the values of score function are different, there are some differences compared to the ranking result derived by our proposed methods.

In the following, we will dedicate to figure out the reasons for those different ranking results.

\subsubsection{Comparison with PFLNWAA and PFLNWGA operator}

In this section, we explore the influence of PFLWMM and PFLWDMM operators on the ranking results of DM schemes. Exploiting PFLNWAA and PFLNWGA operator in Ashraf et al. (2018) to calculate the above example as a comparison. The comparison aspects including the score function and ranking results. The comparison results are shown in Table 5 and Fig. 2.

The operator aggregation method for calculating the ranking of schemes is different from the PFLNWAA and
PFLNWGA operators Ashraf et al. (2018). This is because this paper uses the language scaling function to ensure that the calculation results fall within the pre-defined language interval, and fully explore the influence of the relationship between attributes. The results in the table have become a strong support for the method in this paper. From this new perspective, the results of the method proposed in this article are more reasonable and credible.

\subsubsection{Comparison with A-PFLWAA operator}

In this section, we explore the influence of A-PFLWAA operator on the ranking results of DM schemes. Exploiting A-PFLWAA operator in Liu and Zhang (2018) to calculate the above example as a comparison. The comparison aspects including the score function and ranking results. The comparison results are shown in Table 5 and Fig. 2.

It can be seen that the operator aggregation method for calculating the ranking of the scheme is different from the PFLNWGA operator Liu and Zhang (2018). This is because the method proposed in this paper transforms the subscript calculation of LTs into the corresponding semantic calculation, which can easily give a reasonable explanation to the meaning of the operation rules and avoid erroneous results. Liu and Zhang (2018) exploited Archimedean t-norm and s-norm defined PFLV general operating rules still have limitations, because the calculation of the language term in PFLS is directly based on the subscript of LTs, this method cannot reasonable explanation subscript calculation.

\subsubsection{Influence of parameter $Q$ on the decision-making results}

In this section, we take diverse values to $Q$ in the PFLWMM and PFLWDMM operators to obtain the score function and ranking order. Exploiting PFLNWAA, PFLNWGA and APFLWAA operator operators in Ashraf et al. (2018); Liu and Zhang (2018) to calculate the above example as a comparison. The comparison aspects including the semantics of linguistic terms, the relationship of multiple attributes and information aggregation. The comparison results are shown in Table 6 and Fig. 3.

From Table 6 and Fig. 3, we can easily conclude that the score function values varies with the parameter $Q$ changes. When setting different $\mathrm{Q}$ except $Q=(1,0,0,0)$, the respective overall ranking order of PFLWMM and PFLWDMM operator are the same, namely, the order of PFLWMM is $A_{4} \succ A_{2} \succ A_{1} \succ A_{3}$, and the order of PFLWDMM is $A_{4} \succ A_{3} \succ A_{2} \succ A_{1}$. It is sufficient to verity the effectiveness of proposed methods. What's more, it is obvious to see that the score function values of each alternative decrease with increasing of the interrelationship of the input attribute in PFLWMM operator, while the score function values of 
Table 5 Ranking results by different methods

\begin{tabular}{|c|c|c|}
\hline Aggregation operator & Score function $E\left(\mathrm{~h}_{i}\right)$ & Ranking results \\
\hline$P F L N W A A[22]$ & $E\left(A_{1}\right)=1.939, E\left(A_{2}\right)=2.140 E\left(A_{3}\right)=2.046, E\left(A_{4}\right)=2.995$ & $A_{4} \succ A_{2} \succ A_{3} \succ A_{1}$ \\
\hline$P F L N W G A[22]$ & $E\left(A_{1}\right)=3.065, E\left(A_{2}\right)=3.163 E\left(A_{3}\right)=2.874, E\left(A_{4}\right)=3.660$ & $A_{4} \succ A_{2} \succ A_{1} \succ A_{3}$ \\
\hline$A-P F L W A A[23]$ & $\begin{array}{l}E\left(A_{1}\right)=1.913, E\left(A_{2}\right)=2.263 E\left(A_{3}\right)=1.731, E\left(A_{4}\right)=3.271 \\
\quad(\text { suppose } g(x)=-\log x)\end{array}$ & $A_{4} \succ A_{2} \succ A_{1} \succ A_{3}$ \\
\hline$P F L W M M$ & $\begin{array}{l}E\left(A_{1}\right)=1.809, E\left(A_{2}\right)=1.985 E\left(A_{3}\right)=1.354, E\left(A_{4}\right)=2.310 \\
\quad(\text { suppose } P=(0.2,0.3,0.2,0.3)\end{array}$ & $A_{4} \succ A_{2} \succ A_{1} \succ A_{3}$ \\
\hline$P F L W D M M$ & $\begin{array}{l}E\left(A_{1}\right)=2.074, E\left(A_{2}\right)=2.452 E\left(A_{3}\right)=2.486, E\left(A_{4}\right)=3.069 \\
\quad \text { suppose } P=(0.2,0.3,0.2,0.3)\end{array}$ & $A_{4} \succ A_{3} \succ A_{2} \succ A_{1}$ \\
\hline
\end{tabular}

Fig. 2 Ranking results by different methods
Table 6 Ranking results by utilizing different values of $Q$ in the proposed operators

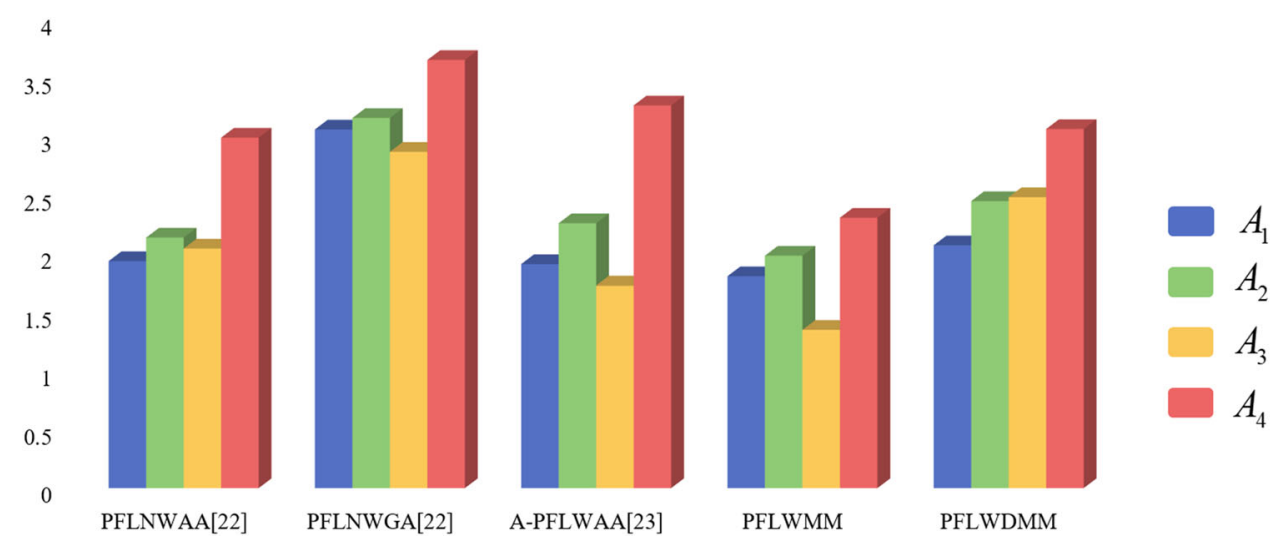

\begin{tabular}{lllllll}
\hline Parameter vector $Q$ & Operator & \multicolumn{3}{c}{ Score values of alternatives } & \multicolumn{2}{l}{ Ranking results } \\
& & $A_{1}$ & $A_{2}$ & $A_{3}$ & $A_{4}$ & \\
\hline$Q=(1,0,0,0)$ & PFLWMM & 2.016 & 2.304 & 2.429 & 3.139 & $A_{4} \succ A_{3} \succ A_{2} \succ A_{1}$ \\
& PFLWDMM & 1.757 & 1.901 & 1.294 & 2.438 & $A_{4} \succ A_{2} \succ A_{1} \succ A_{3}$ \\
$Q=(1,1,0,0)$ & PFLWMM & 1.898 & 2.142 & 1.777 & 2.682 & $A_{4} \succ A_{2} \succ A_{1} \succ A_{3}$ \\
& PFLWDMM & 2.090 & 2.149 & 2.151 & 2.694 & $A_{4} \succ A_{3} \succ A_{2} \succ A_{1}$ \\
$Q=(1,1,1,0)$ & PFLWMM & 1.845 & 2.053 & 1.487 & 2.417 & $A_{4} \succ A_{2} \succ A_{1} \succ A_{3}$ \\
& PFLWDMM & 2.023 & 2.308 & 2.367 & 2.884 & $A_{4} \succ A_{3} \succ A_{2} \succ A_{1}$ \\
$Q=(1,1,1,1)$ & PFLWMM & 1.804 & 1.980 & 1.341 & 2.299 & $A_{4} \succ A_{2} \succ A_{1} \succ A_{3}$ \\
& PFLWDMM & 2.076 & 2.442 & 2.502 & 3.071 & $A_{4} \succ A_{3} \succ A_{2} \succ A_{1}$ \\
$Q=(2,2,2,2)$ & PFLWMM & 1.804 & 1.980 & 1.342 & 2.299 & $A_{4} \succ A_{2} \succ A_{1} \succ A_{3}$ \\
& PFLWDMM & 2.076 & 2.461 & 2.502 & 3.072 & $A_{4} \succ A_{3} \succ A_{2} \succ A_{1}$ \\
$Q=(2,0,0,0)$ & PFLWMM & 2.103 & 2.394 & 2.727 & 3.300 & $A_{4} \succ A_{2} \succ A_{1} \succ A_{3}$ \\
& PFLWDMM & 1.652 & 1.727 & 1.107 & 2.301 & $A_{4} \succ A_{3} \succ A_{2} \succ A_{1}$ \\
$Q=(3,0,0,0)$ & PFLWMM & 2.188 & 2.474 & 2.980 & 3.428 & $A_{4} \succ A_{2} \succ A_{1} \succ A_{3}$ \\
& PFLWDMM & 1.551 & 1.631 & 0.976 & 2.195 & $A_{4} \succ A_{3} \succ A_{2} \succ A_{1}$ \\
\hline
\end{tabular}

Table 7 The comparisons of different methods

\begin{tabular}{llll}
\hline Methods & $\begin{array}{l}\text { Whether the semantics of } \\
\text { linguistic terms are } \\
\text { considered }\end{array}$ & $\begin{array}{l}\text { Whether the relationship of } \\
\text { multiple attributes is capture }\end{array}$ & $\begin{array}{l}\text { Whether make information } \\
\text { aggregation more flexible } \\
\text { by a parameter or function }\end{array}$ \\
\hline PFLNWAA & No & No & No \\
PFLNWGA & No & No & No \\
A-PFLWAA & No & No & Yes \\
The proposed methods & Yes & Yes & Yes \\
\hline
\end{tabular}



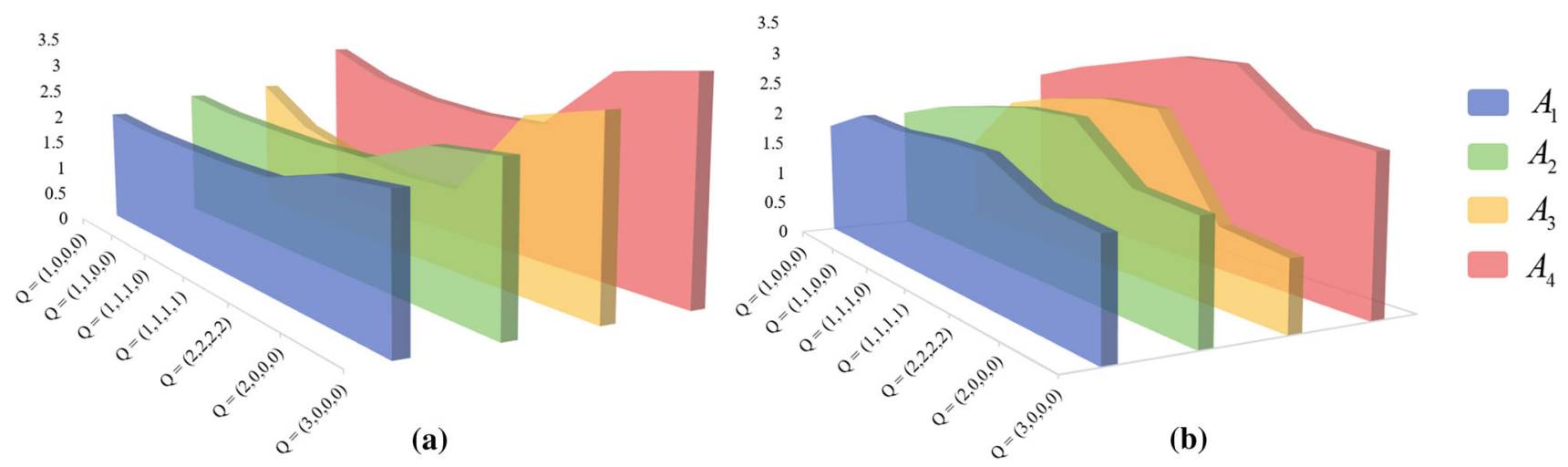

Fig. 3 Ranking results by different methods (a) PFLWMM. (b) PFLWDMM

each alternative increase with increasing of the interrelationship of the input attribute in PFLWDMM operator. Hence, the parameter $Q$ can be regarded as the DM's risk preference, and DM can choose appropriate $Q$ based on actual conditions.

\subsubsection{Further discussion}

To more intuition show the superiority and the typical characteristics of our proposed methods compared with exiting other methods, we conduct a comparative analysis whose features are listed in Table 7.

From Table 7, it is evident that the approaches in Ashraf et al. (2018) fuse picture fuzzy linguistic information through commonly used weighted averaging operator, and the operation process is relatively simple. However, there are some limitations, including the computation results may be beyond the predefined LTSs, and not enough flexibility to face actual situations, etc. In addition, although the methods by Liu and Zhang (2018) can face different conditions by choose diverse function $g(x)$, the interrelationship of input attribute is not considered, because it assumes that all input attributes are independent. Our proposed methods capture the interrelationship of among attributes, but also shows good flexibility and practically by adjusting the values of argument $Q$, namely, the methods we proposed can effectively make up for the shortcomings of above mention methods. Therefore, the proposed methods are more reasonable and flexible than some exiting methods to solve the Picture fuzzy linguistic problems.

\section{Conclusions}

Picture fuzzy language set is an effective tool to express the complex cognitive information in MADM problems. However, the current research results still have many limitations for the MADA problem of the fuzzy set analysis of exploiting PFLS. Specifically, the operational rules and comparison rules of PFLS are not yet complete. The language term part may exceed the upper limit of the redefined $S$, so it is impossible to exploit PFLS to compare and analyze MADM problems with such situations. Moreover, most of the attributes in the MADM problems have correlations, and the current PFLS methods treat the attributes as independent, which may be caused irrational outputs. Committed to solving the above problems, in this article, some PFLVs operating rules are re-improved by introducing LSFs, and then a novel score function and accuracy function are proposed. Then, in order to reasonably deal with the relationship between the attributes, MM and DMM operators are employed to process PFLV, and two aggregation operators are proposed based on the new operation rules, including the Picture Fuzzy Language Weighted MM (PFLWMM) operator and Picture fuzzy language weighted DMM (PFLWDMM) operator. The different properties of those recommended operators are studied. In order to verify the superiority of the proposed operator, two MADM methods based on PFLWDMM and PFLWDMM operators were developed to solve the problem of selecting the design scheme of shared bicycle "Mobike". The results show that the scheme recommendation based on the proposed method is significantly different from other schemes, which shows the effectiveness of the proposed method.

In the future, we will further discuss the new method of considering the MADM problem under the condition of attribute correlation in PFLV, such as from the perspective of fuzzy soft sets and fuzzy soft graphs Akram and Luqman (2020); Adeel et al. (2020). The current method uses MM and DMM operators to process the correlation, and its calculation is slightly more complicated. In addition, it is of great significance to further study the related theories of PFLV and explore their applications in the decision-making of data-driven companies. 
Acknowledgements This work was supported by the Graduate Teaching Reform Research Program of Chongqing Municipal Education Commission (No.YJG212022) and Chongqing Research and Innovation Project of Graduate Students (No. CYS21326).

\section{Declarations}

Conflict of interest The authors declare that they have no conflict of interest.

Ethical statement Articles do not rely on clinical trials.

Human and animal participants All submitted manuscripts containing research which does not involve human participants and/or animal experimentation.

\section{References}

Adeel A, Akram M, Ahmed I, Nazar K (2019) Novel $m$-polar fuzzy linguistic ELECTRE-I method for group decision-making. Symmetry 11(4):471

Adeel A, Akram M, Koam ANA (2019) Group decision-making based on $m$-polar fuzzy linguistic TOPSIS method. Symmetry 11(6):735

Adeel A, Akram M, Yaqoob N, Chammam W (2020) Detection and severity of tumor cells by graded decision-making methods under fuzzy N-soft model. J Intell Fuzzy Syst 39(1):1303-1318

Akram M, Luqman A (2020) Granulation of ecological networks under fuzzy soft environment. Soft Comput 24:11867-11892

Akram M, Adeel A, Al-Kenani AN, Alcantud JC (2021) Hesitant fuzzy N-soft ELECTRE-II model: a new framework for decisionmaking. Neural Comput Appl 33:7505-7520

Akram M, Naz S, Edalatpanah SA, Mehreen R (2021) Group decisionmaking framework under linguistic q-rung orthopair fuzzy Einstein models. Soft Comput. https://doi.org/10.1007/s00500-02105771-9

Akram M, Peng X, Sattar A (2021) A new decision-making model using complex intuitionistic fuzzy Hamacher aggregation operators. Soft Comput 25:7059-7086. https://doi.org/10.1007/s00500021-05658-9

Ashraf S, Mahmood T, Khan Q (2018) Picture fuzzy linguistic sets and their applications for multi-attribute group decision making problems. The Nucl 55(2):66-73

Atanassov KT (1986) Intuitionistic fuzzy sets. Fuzzy Sets Syst 20(1):87-96

Cuong BC, Hai PV (2015) Seventh international conference on knowledge and systems engineering (KSE). IEEE. pp. 132-137

Cuong BC, Kreinovich V (2014) Picture fuzzy sets. J Comput Sci Cybern 30(4):409-420

Gou XJ, Xu ZS, Liao HC (2017) Multiple criteria decision making based on Bonferroni means with hesitant fuzzy linguistic information. Soft Comput 21(21):6515-6529

Herrera F, Herrera-Viedma E, Verdegay JL (1995) A sequential selection propose in group decision making with a linguistic assessment approach. Inform Sci 85:223-239

Herrera F, Herrera-Viedma E, Chiclana F (2001) Multiperson decisionmaking based on multiplicative preference relations. Eur J Op Res 129(2):372-385

Jana C, Senapati T, Pal M, Yager RR (2019) Picture fuzzy Dombi aggregation operators: application to MADM process. Appl Soft Comput J 74:99-109
Ju YB, Ju DW, Wang AH (2020) A note on Picture 2-tuple linguistic aggregation operators in multiple attribute decision making. Soft Comput 24(6):3937-3941

Khalil AM, Li S, Garg H, Li H, Ma S (2019) New operations on intervalvalued picture fuzzy set, interval-valued picture fuzzy soft set and their applications. IEEE Access 7:51236-51253

Li JJ, Wang JQ (2009) The multi-criteria group decision making method based on multi-granularity intuitionistic two semantics. Sci Technol Inform 33:8-9

Liao H, Wu X, Liang X, Yang JB, Xu DL (2018) A continuous intervalvalued linguistic OREST method for multi-criteria group decision making. Knowl Based Syst 153:65-77

Liu PD, Zhang XH (2018) A novel picture fuzzy linguistic aggregation operator and its application to group decision-making. Cognit Comput 10242-259:249-259

liu PD, Khan Q, Ye J, Mahmood T (2020) Group decision-making method under hesitant interval neutrosophic uncertain linguistic environment. Int J Fuzzy Syst 20(7):2337-2353

Muirhead RF (1902) Some methods applicable to identities and inequalities of symmetric algebraic functions of $n$ letters Proceedings of the Edinburgh Mathematical Society. 21: 144-162

Peng XD, Yang Y (2016) Multiple attribute group decision making methods based on Pythagorean fuzzy linguistic set. Comput Eng Appl 52:50-55

Qin JD, Liu XW (2016) 2-tuple linguistic Muirhead mean operators for multiple attribute group decision making and its application to supplier selection. Kybernetes 45(1):2-29

Qiyas M, Abdullah S, Ashraf S, Aslam M (2020) Utilizing linguistic picture fuzzy aggregation operators for multiple-attribute decisionmaking problems. Int J Fuzzy Syst 22(1):310-320

Rong Y, liu Y, Pei Z, (2020) Complex q-rung orthopair fuzzy 2-tuple linguistic Maclaurin symmetric mean operators and its application to emergency program selection. Int J Intell Syst 35(11):17491790

Si A, Das S, Kar S (2021) Picture fuzzy set-based decision-making approach using Dempster-Shafer theory of evidence and grey relation analysis and its application in COVID-19 medicine selection. Soft Comput. https://doi.org/10.1007/s00500-021-05909-9

Singh P (2015) Correlation coeffificients for picture fuzzy sets. J Intell Fuzzy Syst 27:2857-2868

Singh V, Yadav SP, Mesiar R (2020) Information measures in Atanassov's intuitionistic fuzzy environment and their application in decision making. IEEE Trans Fuzzy Syst 28(11):2905-2917

Son LH (2015) DPFCM. A novel distributed picture fuzzy clustering method on picture fuzzy sets. Exp Syst Appl 42(1):51-66

Wang ZJ (2020) A representable uninorm-based intuitionistic fuzzy analytic hierarchy process. IEEE Trans Fuzzy Syst 28(10):25552569

Wang JQ, Wu JT, Wang J, Zhang HY, Chen XH (2014) Interval-valued hesitant fuzzy linguistic sets and their applications in multi-criteria decision-making problems. Inform Sci 288:55-72

Wang L, Zhang HY, Wang JQ, Wu GH (2020) Picture fuzzy multicriteria group decision-making method to hotel building energy efficiency retrofit project selection. Rairo-Op Res 54(1):211-229

Wei GW (2017) Picture fuzzy aggregation operators and their application to multiple attribute decision making. J Intell Fuzzy Syst 33(2):713-724

Wei GW (2018) Picture fuzzy hamacher aggregation operators and their application to multiple attribute decision making. Fundamenta Informat 157(3):271-320

Xia MM, Xu ZS, Zhu B (2013) Geometric Bonferroni means with their application in multi-criteria decision making. Knowl Based Syst 40:88-100

Xian SD, Xue WT, Zhang JF, Yin YB, Xie Q (2015) Intuitionistic fuzzy linguistic induced ordered weighted averaging operator for group 
decision making. Int $\mathrm{J}$ Uncertain Fuzziness Knowl Based Syst 23:627-648

$\mathrm{Xu}$ ZS (2004) Uncertain linguistic aggregation operators based approach to multiple attribute group decision making under uncertain linguistic environment. Inform Sci 168(1-4):171-184

$\mathrm{Xu}$ ZS (2012) Linguistic decision making: theory and methods. Springer, Berlin Heidelberg

Yang L, Wu XH, Qian J (2020) A novel multicriteria group decisionmaking approach with hesitant picture fuzzy linguistic information. Math Prob Eng. https://doi.org/10.1155/2020/6394028
Zadeh LA (1965) Fuzzy sets. Inform Control 8(3):338-356

Zadeh LA (1975) The concept of a linguistic variable and its application to approximate reasoning-III. Inform Sci 9(1):43-80

Zhang XY, Wang J, Wang JQ, Hu JH (2020) A revised picture fuzzy linguistic aggregation operator and its application to group decision-making. Cognit Comput 12(5):1070-1082

Zhang SQ, Wei GW, Alsaadi FE, Hayat T, Wei C, Zhang ZP (2020) MABAC method for multiple attribute group decision making under picture 2-tuple linguistic environment. Soft Comput 24(8):5819-5829

Publisher's Note Springer Nature remains neutral with regard to jurisdictional claims in published maps and institutional affiliations. 